\title{
Brain-inspired memristive neural networks for unsupervised learning
}

\author{
D. Ielmini and V. Milo
}

\begin{abstract}
Memristive devices, such as resistive switching memory (RRAM) and phase change memory (PCM), show variable resistance which can mimic the synaptic plasticity in the human brain. This fascinating analogy has provided the inspiration for many recent research advances, involving memristive devices and their use as artificial electronics synapses in neuromorphic circuits with learning capability. In particular, RRAM-based artificial synapses are extremely promising in terms of area efficiency, low power consumption, and flexibility of design which pave the way for spiking neural networks that perform and behave like the human brain. This chapter will review the state of the art about the design and development of memristive neural networks for unsupervised learning. First, the optimization of RRAM devices for synaptic applications will be discussed, and a novel RRAM device with improved resistance window and controllability of resistance will be introduced. Then, a hybrid CMOS/memristive synaptic circuit will be shown to carry out learning tasks via the spike-timing dependent plasticity (STDP), which is one of the learning rules in biological synapses. Finally, the neural networks based on RRAM synapses will be reviewed, covering both feed-forward networks and recurrent networks. In both cases, the network displays unsupervised learning of input patterns, which can be stored, recognized, or even reconstructed by the network, thus highlighting the wealth of potential promising applications for memristive networks with synaptic plasticity.
\end{abstract}

D. Ielmini

Dipartimento di Elettronica, Informazione e Bioingegneria and Italian Universities Nanoelectronics Team (IU.NET), Politecnico di Milano, Piazza L. da Vinci 32, 20133 Milan, Italy.

e-mail: daniele.ielmini@polimi.it

V. Milo

Dipartimento di Elettronica, Informazione e Bioingegneria and Italian Universities Nanoelectronics Team (IU.NET), Politecnico di Milano, Piazza L. da Vinci 32, 20133 Milan, Italy.

e-mail: valerio.milo@polimi.it 


\section{Introduction}

Resistive switching memory (RRAM) is a 2-terminal element which can change its resistance $R$, or conductance $G=1 / R$, via the application of a voltage signal $[1,2,3,4,5]$. Resistive switching effects in metal oxides were originally discovered in the 1960s $[6,7,8]$, then later studied for potential application in nonvolatile memory devices $[9,10,11,12]$. Today, the research on RRAM for electronic storage has been mostly transferred to industrial development of storage-class memory [13] and embedded memory for Internet of Things (IoT) [14]. On the other hand, RRAM devices have stimulated an increasing interest for the development of artificial synapses in neural networks. In fact, a RRAM device shows controllable conductance change in both binary (digital) and multilevel (analog) mode, thus being possibly implemented as a plastic neuromorphic synapse similar to biological synapses in the human brain. In this frame, RRAM can be viewed as a memristive device, i.e., a variable resistive element which can change its conductance in response to the applied voltage $[15,16]$. Engineering RRAM synapses with tunable weight has become a grand challenge toward the development of neuromorphic circuits capable of learning via synaptic plasticity.

The current focus of the research on memristive synapses includes 2 types of neural networks. First, deep neural networks (DNNs) with multi-layer perceptron (MLP) structure show promising properties of inference after supervised training [17]. Typically, DNNs rely on non-spiking neurons and weight update based on supervised learning algorithms such as the backpropagation scheme [18]. In backpropagation, the MLP output is compared with the ideal solution, which is carried by labels, and the resulting difference, i.e., the error, is back-propagated to proportionally update all synapses in the network, until the recognition accuracy is improved above a certain threshold. DNNs have been demonstrated for supervised learning with various types of memristive synapses including PCM [19] and RRAM [20, 21]. In a DNN, memristive devices offer the possibility to store a multilevel weight in a nanoscale element, thus allowing to reduce the circuit area of the synaptic array. Most importantly, the matrix-vector multiplication (MVM) is carried out physically within the memristor array thanks to the Kirchhoff's law and the Ohm's law, instead of relying on extensive multiply-accumulate (MAC) operations [22]. The acceleration of MVM is however contrasted by inevitable approximation related to the imperfect programming of memristive devices and the time-dependent fluctuations arising after programming, e.g., related to drift in PCM [23] and noise in RRAM [24]. Finally, the non-linearity of the resistance change process in RRAM and PCM is an additional challenge that severely degrades the learning accuracy of DNNs [25, 26, 27]. On the other hand, the spiking neural network (SNN) is viewed as a suitable solution for event-driven processing, similar to the human brain, thus potentially resulting in higher energy efficiency, larger density of information, and higher computing functionality $[28,29]$. SNNs are also more suitable to achieve unsupervised learning, where patterns are received and stored in the network without labels, which is one of the most general learning mode of the human brain. To achieve unsupervised learning in absence of labels, the delay between spikes is used as feedback informa- 
tion across the SNN layers, through the spike-timing dependent plasticity (STDP) rule [30, 31, 32, 33, 34]. Designing memristive synapses capable of STDP, and designing SNN architectures able to replicate computation primitives of the brain is among the greatest challenges for memristive neuromorphic engineering.

This chapter provides an overview of memristive SNNs capable of unsupervised learning, focusing on RRAM-based synapses at the level of device, synaptic circuit, and memristive SNNs. First, the device optimization strategy for synaptic application will be discussed, with reference to a novel RRAM technology based on $\mathrm{SiO}_{x}$ for improved on/off ratio. Then, a circuit proposal for STDP synapses using RRAMs and PCMs will be described, showing experimental demonstration of individual synaptic circuit blocks and their characteristics. Finally, the SNN architectures will be analyzed, covering both feed-forward and recurrent networks for unsupervised learning of patterns. Pattern learning, storage, recognition, reconstruction and association will be demonstrated in SNNs by circuit simulation and experimental demonstration with physical memristive RRAM synapses. The results pave the way for brain-inspired SNNs capable of unsupervised learning, inference and planning.

\section{RRAM devices}

To develop SNNs capable of learning with RRAM synapses, the RRAM device must be optimized to fit the specifications of both nonvolatile storage and in-memory computing. This challenging task is accomplished by a detailed understanding, modeling, and engineering of RRAM materials with emphasis on programming performances (energy consumption, programming voltage, set/reset speed), reliability characteristics (retention time, endurance, variability and noise) and scaling. To enable such a broad landscape of device properties, materials must be carefully selected and combined in the RRAM stack.

A RRAM device is operated by the formation and disconnection of a conduc-

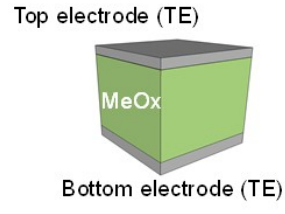

(a) Initial state

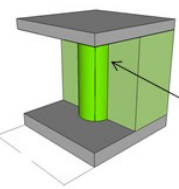

(b) Set state

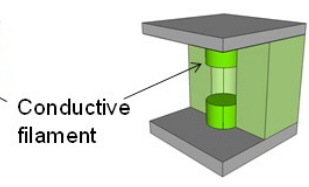

(c) Reset state

Fig. 1 RRAM device states. (a) The device features a dielectric layer, such as a binary metal oxide $\mathrm{MeO}_{x}$. (b) After forming, the device is left in a set state, or LRS, due to the presence of a CF shunting the TE and BE. (c) The application of a reset pulse leads to the disconnection of the CF thus resulting in the reset state, or HRS. Transition back to the LRS is possible via the application of a set pulse. Reprinted with permission from [35]. Copyright 2014 Wiley and Sons. 
tive filament $(\mathrm{CF})$, or percolation path, along an insulating material, as depicted in Fig. 1 [35]. Initially, the device features a dielectric layer, e.g., a metal oxide $\mathrm{MeO}_{x}$ in Fig. 1a. The switching operation in the dielectric is initiated by a forming operation, where a dielectric breakdown is first induced across $\mathrm{MeO}_{x}$ to generate a sufficient amount of defects, such as oxygen vacancies, or excess metallic impurities of the constituent metal Me or originating from the electrodes. The oxygen concentration $\mathrm{x}$ in the metal oxide is usually kept below the stoichiometric value, e.g., $\mathrm{x}$ is generally lower than 2 in $\mathrm{HfO}_{x}$, to allow a certain concentration of defects to be present in the pristine oxide layer and facilitate oxide breakdown at relatively low voltage. After forming (Fig. 1b), the device is in the so-called set state, or low resistance state (LRS), due to the CF shunting the top electrode (TE) and the bottom electrode (BE). The application of a reset operation leads to the disconnection of the $\mathrm{CF}$, and the corresponding transition to the so-called reset state, or high resistance state (HRS), as shown in Fig. 1c. The CF is then recovered by a set operation which leads back to the LRS. In bipolar switching RRAM technology, which constitutes the large majority of RRAM devices currently studied by academic and industrial research, the set and reset operations consist of the application of voltage sweeps or pulses with opposite polarities, e.g., positive voltage for the set transition and negative voltage for the reset transition, where the voltage is assumed to be applied to the TE.

RRAM devices can be generally distinguished in 2 technologies, namely RRAM relying on the resistive switching of metal oxides, such as $\mathrm{HfO}_{x}[36,37,38,39]$, $\mathrm{TiO}_{x}[40,41]$ and $\mathrm{TaO}_{x}[42,43]$, and RRAM based on the electrochemical reaction and migration of cations from an active electrode, also known as conductive bridge memory (CBRAM) [44, 45, 46]. In the latter case, the dielectric material can be either a metal oxide, or another insulating layer, also referred to as electrolyte, such as GeSe [44], $\mathrm{GdO}_{x}$ [45], $\mathrm{GeS}_{2}$ [46, 47], $\mathrm{ZrO}_{x}$ [48] and $\mathrm{Al}_{2} \mathrm{O}_{3}$ [49]. The active electrode generally consists of $\mathrm{Ag}$ [47], $\mathrm{Cu}[13,44,50]$, or their compounds, such as CuTe [45]. The resistance window between HRS and LRS is generally larger in CBRAM-type devices compared to oxide-based RRAM [51], which enables a higher immunity to switching variations $[52,53]$ and current fluctuations [24, 54, 55], constituting a significant concern for nanoscale RRAM reliability. On the other hand, CBRAM suffers from a relatively short retention time, as demonstrated by several reports of volatile CBRAM where the retention time is well below $1 \mathrm{~s}[47,56,57,58,59,60,61]$.

To achieve a good device stability and a high resistance window, the promising properties of oxide- and CBRAM-type RRAM devices should be combined. To this purpose, a dielectric material with high band gap should be adopted, to enable high resistance of the HRS. To enable high resistance window and a high stability at the same time, the TE should be reasonably active, although avoiding the choice of $\mathrm{Ag}$ and $\mathrm{Cu}$ which may lead to volatile switching behavior. Finally, the BE material should be inert to prevent set transition under negative applied voltage during the reset operation [39].

Based on these considerations, a novel RRAM technology was recently proposed, which combines a $\mathrm{SiO}_{x}$ dielectric layer, a Ti-based TE and a C-based $\mathrm{BE}$, as de- 
(a)

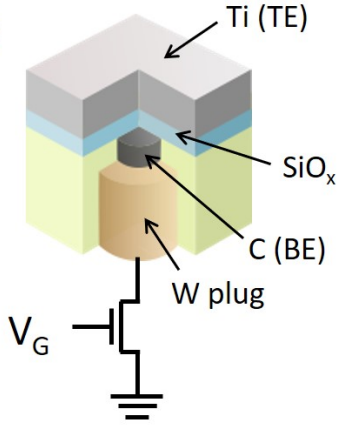

(b)

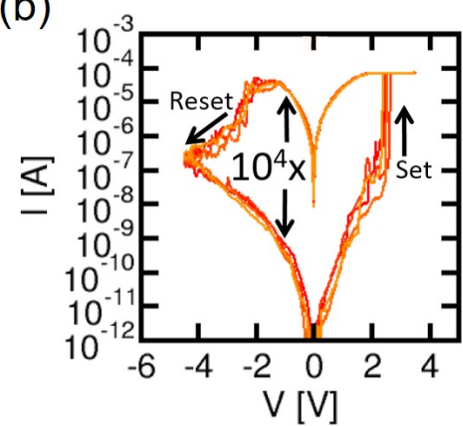

Fig. $2 \mathrm{SiO}_{x}$-based RRAM device. (a) Device stack structure and (b) measured $I-V$ curves, evidencing set and reset transitions at positive and negative voltages, respectively. A large resistance window of about $10^{4}$ is obtained thanks to the high band gap of the $\mathrm{SiO}_{x}$ dielectric layer and the complete dissolution of the Ti-based CF in the HRS. Reprinted with permission from [58]. Copyright 2016 IEEE.

picted in Fig. 2a [58, 62]. The Ti cap serves as defect-injecting reservoir layer during set, where Ti cations migrate into the $\mathrm{SiO}_{x}$ to form the CF under a positive applied voltage [63, 64]. The application of a negative voltage leads to migration of Ti cations back to the TE, with no further defect injection from the BE due to the inert quality of the graphitic $\mathrm{C}$ layer. The $\mathrm{BE}$ is also confined into a 70-nm plug to enable evaluation of the forming, switching, and reliability properties at the nanoscale. The $\mathrm{SiO}_{x}$ layer was deposited by e-beam evaporation from a $\mathrm{SiO}$ source, thus $\mathrm{x}$ should be around 1 in the device stack.

Fig. $2 \mathrm{~b}$ shows the measured $I-V$ curves of a $\mathrm{SiO}_{x}$-RRAM device connected to an integrated field-effect transistor (FET) to control the maximum current during the set transition, also referred to as compliance current $\mathrm{I}_{C}$ [63]. In the figure, $\mathrm{I}_{C}$ was limited to about $70 \mu \mathrm{A}$ to study the device operation under relatively low current consumption. The $I-V$ curves show set transition for positive voltage and reset transition at negative voltage, with a resistance window of about $10^{4}$ between the LRS and HRS, despite the relatively high resistance of the LRS due to the low $\mathrm{I}_{C}$. The high resistance window is due to the high band gap of the $\mathrm{SiO}_{x}$ layer, combined with the CBRAM-type switching mode of the device, where Ti defects are almost completely removed from the $\mathrm{SiO}_{x}$ layer after reset, thus enabling a relatively high resistance of the HRS. The abrupt set transition to the LRS reveals the sudden formation of the $\mathrm{CF}$, where more defects introduced into the $\mathrm{SiO}_{x}$ enhance the electric field and Joule heating, thus inducing the self-accelerated migration of defects $[65,66]$. On the other hand, the reset transition shows more gradual increase of resistance, as the migration of defects causes an increase in the width of the depleted gap, thus reducing the electric field and Joule heating. As a result, once the reset transition has started, more voltage is needed to further promote migration of defects, which is at the basis of the gradual drop of current in the reset transition of 
(a)

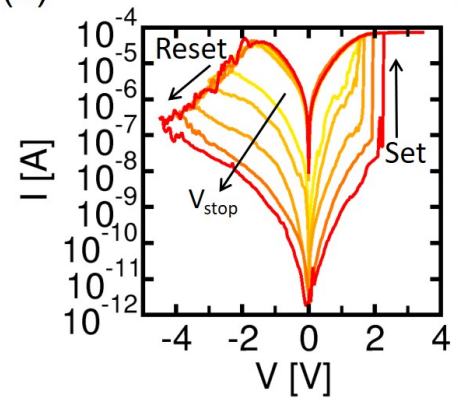

(b)

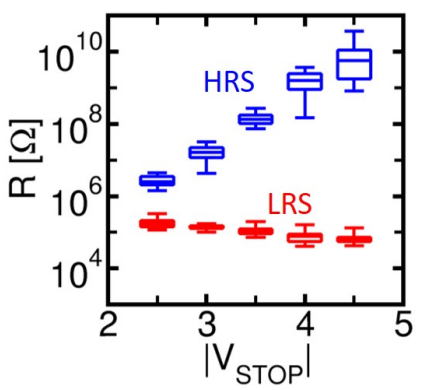

Fig. 3 Control of resistance window in $\mathrm{SiO}_{x}$-based RRAM. (a) Measured $I-V$ curves at increasing $\mathrm{V}_{\text {stop }}$ and (b) corresponding resistance for HRS and LRS as a function of $\mathrm{V}_{\text {stop }}$, for $\mathrm{I}_{C}=70 \mu \mathrm{A}$. The resistance window controllably increases with $\mathrm{V}_{\text {stop }}$, thus enabling multilevel memory operation and gradual synaptic depression. Reprinted with permission from [58]. Copyright 2016 IEEE.

Fig. 2b [66]. Note that the abrupt set transition is potentially interesting for digital memory and logic computing applications [67], whereas synaptic potentiation generally requires gradual increase of the conductance, for progressive STDP and fine updating of the synaptic weights in DNNs [25, 26, 27]. Gradual depression is however possible thanks to the negative feedback of the reset transition, thus enabling the use of the $\mathrm{SiO}_{x}$ RRAM in DNNs for supervised training by backpropagation algorithm.

The gradual reset transition allows to tune the resistance of the HRS, by controlling the width of the depleted gap during reset [67]. This is shown in Fig. 3a, where the HRS resistance at the end of the reset transition increases with the maximum voltage $\mathrm{V}_{\text {stop }}$ applied in the reset sweep. The controllable HRS enables multilevel operation of the $\mathrm{SiO}_{x}$ RRAM, which enhances the scalability of the memory device by allowing for the storage of more than one single bit within a physical memory cell $[68,69,70,71]$. Note that the increase of HRS is reflected by the corresponding increase of the set voltage $\mathrm{V}_{\text {set }}$, marking the set transition under positive applied voltage. This can be explained by the relationship between $\mathrm{V}_{\text {set }}$ and the depleted width $\Delta$ in the HRS, where an increasing $\Delta$ leads to a decreasing field across the depleted gap for a given voltage, thus requiring a larger $\mathrm{V}_{\text {set }}$ to reach the critical field for inducing the set transition by defect migration across the depleted gap [65]. The controllable HRS also allows to tune the resistance window which increases with $\mathrm{V}_{\text {stop }}$ as shown in Fig. 3b. The slight decrease of the LRS resistance with $\mathrm{V}_{\text {stop }}$ can be explained by the higher average field along the depleted gap for high HRS resistance [58, 62]. The $\mathrm{SiO}_{x}$ RRAM also demonstrates high cycling endurance of almost $10^{8}$ cycles, low cycle-to-cycle switching variations, and an excellent stability at elevated temperature, where both HRS and LRS show negligible variations for annealing at $260^{\circ} \mathrm{C}$ for 1 hour [58, 62]. Overall, these favorable properties make $\mathrm{SiO}_{x}$ 


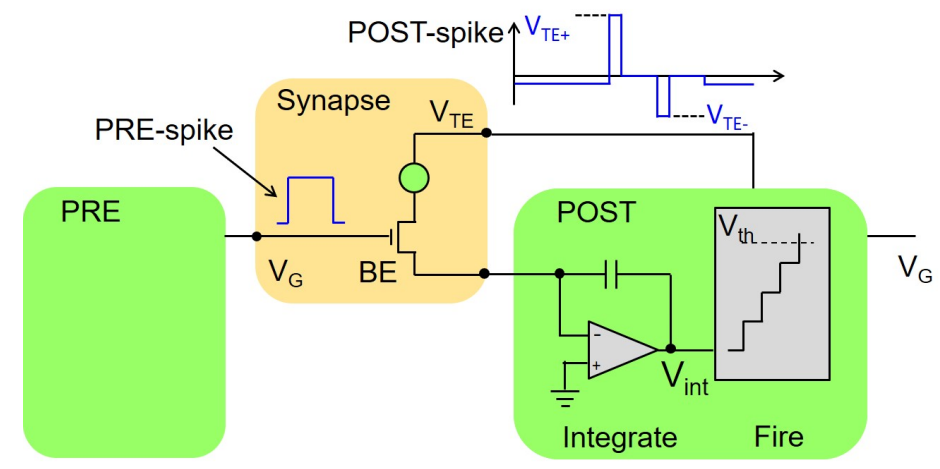

Fig. 4 Sketch of a fundamental circuit block in a feed-forward neural network including a presynaptic neuron (PRE) and a post-synaptic neuron (POST) connected by a resistive synapse with a 1T1R structure. As a spike is generated by the PRE, a current spike is activated across the synapse leading to an increase of $\mathrm{V}_{\text {int }}$ within the POST. As $\mathrm{V}_{\text {int }}$ exceeds the internal threshold $\mathrm{V}_{t h}$ for fire, a backward spike is applied to the TE of the 1T1R synapse, causing the weight update according to STDP. Adapted with permission from [91]. Copyright 2016 IEEE.

RRAM a promising technology for nonvolatile memory and in-memory computing, including neuromorphic memristive networks.

\section{RRAM synapses}

Brain-inspired neuromorphic networks rely on synaptic plasticity according to biological learning rules, such as STDP and spike-rate dependent plasticity (SRDP), to emulate human-brain functionalities including visual/auditory pattern learning [72, $73,74,75,76,77,78,79,80,81,82,83,84,85,86,87,88]$ and pattern classification $[89,90]$. The synaptic plasticity can be implemented at circuit level by combining a RRAM device with a FET in the so-called one-transistor/one-resistor (1T1R) structure, as shown in Fig. 4. Here, the 1T1R synapse is shown as a connecting element between a pre-synaptic neuron (PRE) and a POST-synaptic neuron (POST) $[83,90,91]$. The PRE is connected to the gate of the FET in the 1T1R synapse, while the POST receives the synaptic current from the BE while controlling the voltage at the TE of the 1T1R synapse. The operation of the 1T1R synapse can be understood as follows: as the PRE generates a positive voltage spike, the FET acts as a pass-transistor enabling a synaptic current proportional to the RRAM synaptic conductance. The current spike enters the POST via the BE which can collect incoming currents from several synaptic channels, as in the ideal McCullochPitts (MCP) neuron scheme [92]. The currents are integrated in the integrate-and-fire POST circuit, eventually leading to a fire event as the integral signal $\mathrm{V}_{\text {int }}$ reaches the threshold $\mathrm{V}_{t h}$. At fire, the POST generates a spike toward the next layer of neurons, 
(a)

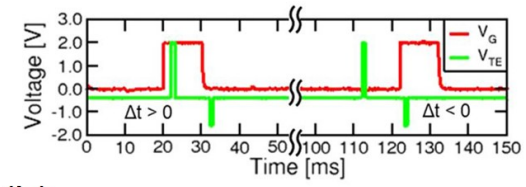

(b)

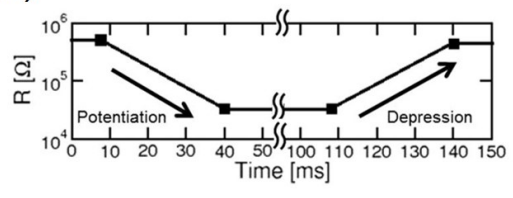

(c)

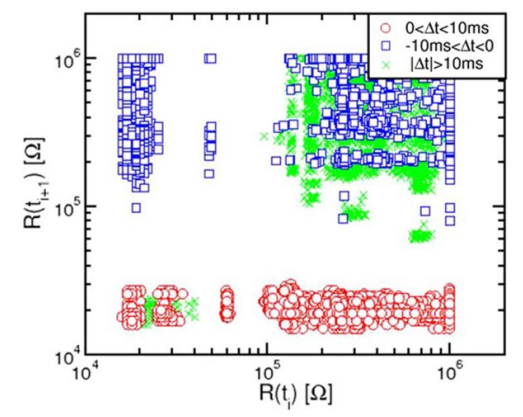

Fig. 5 (a) PRE and POST voltage waveforms applied to the gate and the TE, respectively, for the cases of positive delay (left) and negative delay (right). (b) If the PRE spike occurs before the POST spike $(\Delta t>0)$, the resistance decreases due to the positive TE spike causing set transition, or synaptic potentiation. Otherwise, if the PRE spike occurs after the POST spike $(\Delta t<0)$, the resistance increases due to the negative TE spike causing reset transition, or synaptic depression. (c) Correlation plot of the RRAM resistance $\mathrm{R}\left(\mathrm{t}_{i+1}\right)$ at epoch $\mathrm{t}_{i+1}$ as a function of the RRAM resistance $\mathrm{R}\left(\mathrm{t}_{i}\right)$ at epoch $\mathrm{t}_{i}$ for variable $\Delta \mathrm{t}$, corresponding to the cases of potentiation, depression, and no change of weight because of excessive delay. Reprinted from [87], which is licensed under a Creative Commons Attribution 4.0 International License.

and additionally applies a feedback spike to the synapse TE. The feedback spike consists of the sequence of a positive pulse and a negative pulse, which can induce a weight update depending on the relative timing with the PRE spike, as shown in Fig. 5a. If the PRE spike shortly precedes the POST spike $(0<\Delta t<10 \mathrm{~ms})$, the resulting overlap between the PRE spike and the positive pulse in the POST spike causes set transition, as the positive applied voltage is larger than the set volt-
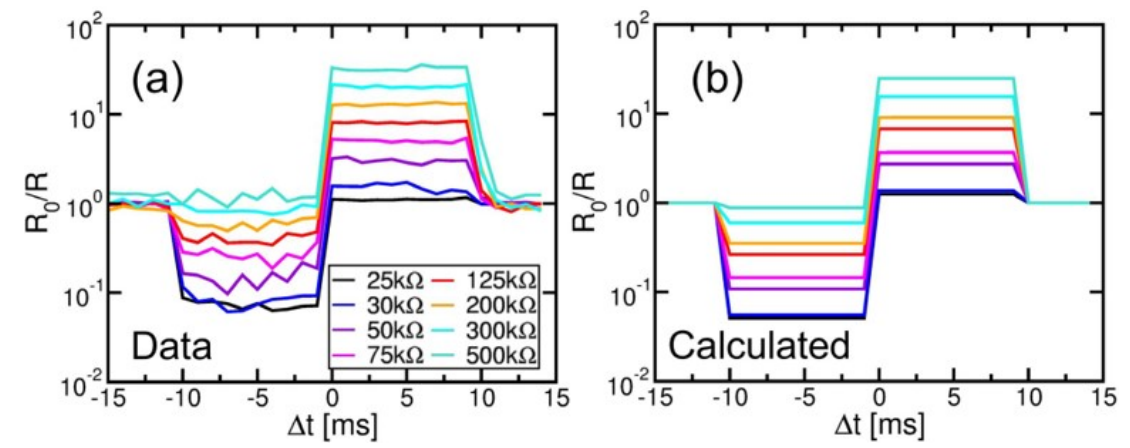

Fig. 6 (a) Measured and (b) calculated STDP characteristics indicating the relative change of resistance $\mathrm{R}_{0} / \mathrm{R}$ as a function of $\Delta \mathrm{t}$ for variable initial resistance states $\mathrm{R}_{0}$, from full LRS $\left(\mathrm{R}_{0}=25 \mathrm{k} \Omega\right.$ ) to full HRS $\left(\mathrm{R}_{0}=500 \mathrm{k} \Omega\right.$ ). Reprinted with permission from [83]. Copyright 2016 IEEE. 
age $\left(V_{T E+}>V_{\text {set }}\right)$, hence causing synaptic potentiation. On the other hand, if the PRE spike shortly follows the POST spike $(-10 m s<\Delta t<0)$, the overlap between the PRE spike and the negative pulse in the POST spike causes reset transition, as the negative applied voltage is larger (in absolute value) than the reset voltage $\left(V_{T E-}<V_{\text {reset }}\right)$, hence causing synaptic depression. Fig. 5b shows the measured resistance before/after the application of spikes in the Fig. 5a, showing that the RRAM device undergoes a set process (synaptic potentiation) for $0<\Delta t<10 \mathrm{~ms}$, whereas the RRAM undergoes a reset process (synaptic depression) for $-10 \mathrm{~ms}<\Delta t<0$.

Figure $5 \mathrm{c}$ shows the correlation plot of the resistance $\mathrm{R}\left(\mathrm{t}_{i+1}\right)$ measured after the spike application as a function of $\mathrm{R}\left(\mathrm{t}_{i}\right)$ measured before the spike application, for variable $\Delta \mathrm{t}$ [87]. Under potentiation condition, namely for positive delay satisfying $0<\Delta t<10 \mathrm{~ms}$, a RRAM prepared in HRS undergoes a set transition to the LRS, whereas if the RRAM device is initially in LRS, no resistance variation occurs because the RRAM is already at its minimum resistance state [87, 93]. For negative delay satisfying $-10 \mathrm{~ms}<\Delta t<0$, corresponding to the condition for synaptic depression, a resistance transition is activated when the RRAM device is initialized in its LRS. Finally, if $\Delta \mathrm{t}$ assumes values outside the plasticity window $(|\Delta t|>10 \mathrm{~ms})$, the PRE and POST spikes do not overlap, therefore the RRAM resistance does not change. As a result of the full set/reset operations taking place in the plasticity mechanism, the 1T1R synapse only displays HRS and LRS resistive levels, thus evidencing the binary operation of the 1T1R synaptic device due to the relatively abrupt set and reset transitions [87]. Note that more resistance levels can be achieved by timedependent modulation of the PRE and POST spikes in the 2T1R synapse [94]. In this synapse architecture, the waveform of the PRE spike allows for time-dependent potentiation, where a longer $\Delta \mathrm{t}$ corresponds to a smaller conductance due to the lower compliance current during set transition. On the other hand, the waveform of the POST spike allows for time-dependent depression, where a longer $\Delta \mathrm{t}$ corresponds to a smaller resistance due to the lower voltage applied during reset transition [94]. The enhanced functionality comes at the expense of a slightly higher complexity of the $2 \mathrm{~T} 1 \mathrm{R}$ synapse circuit, requiring 2 transistors instead of only one in the 1T1R synapse [91].

To further support the dependence of STDP on initial state in the 1T1R synapse, Fig. 6 shows the measured (a) and calculated (b) STDP characteristics, namely the ratio between the initial resistance $\mathrm{R}_{0}$ and the final resistance after potentiation/depression, as a function of $\Delta \mathrm{t}$ for increasing $\mathrm{R}_{0}$ [83]. Calculations were done based on a compact model for RRAM devices [66] including statistical variability [83]. These results show binary STDP behavior, where the amount of potentiation and depression is a function of $\mathrm{R}_{0}$. The variable change of resistance allows the final resistance to be equal to either HRS or LRS, in strong analogy with biological synapses where the weight update is limited between two boundary states. Fig. 7 further illustrates the three-dimensional (3D) color map of the calculated STDP characteristics, evidencing the increase of potentiation/depression level of $1 T 1 R$ synapse with increasing/decreasing $\mathrm{R}_{0}$ for positive/negative $\Delta \mathrm{t}$ [83]. 


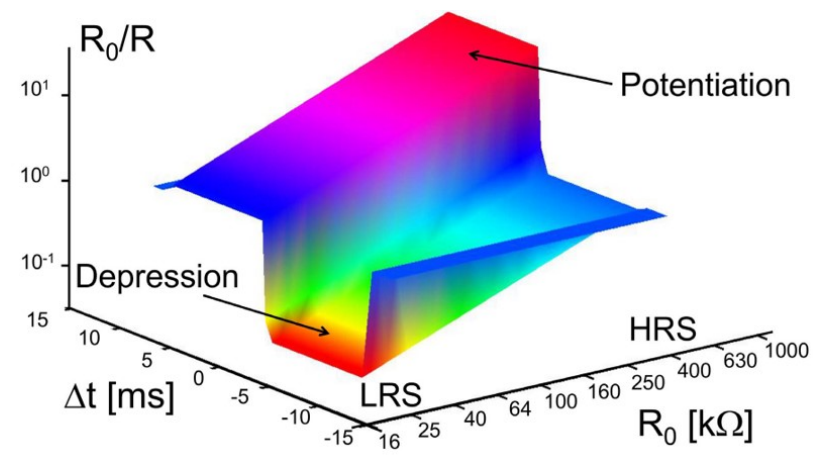

Fig. 7 Three-dimensional (3D) color plot of calculated STDP characteristics shown in Fig. 6. Potentiation and depression are both a function of time delay and the initial synaptic state, resulting in the final state being either HRS or LRS. Reprinted with permission from [83]. Copyright 2016 IEEE.

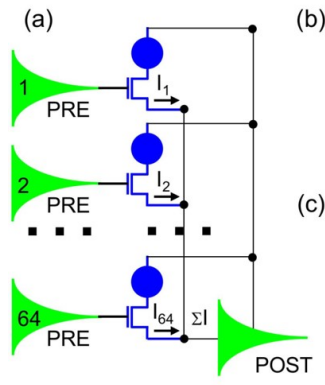

(b)

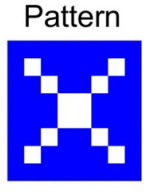

(c)

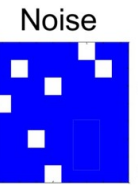

(d)
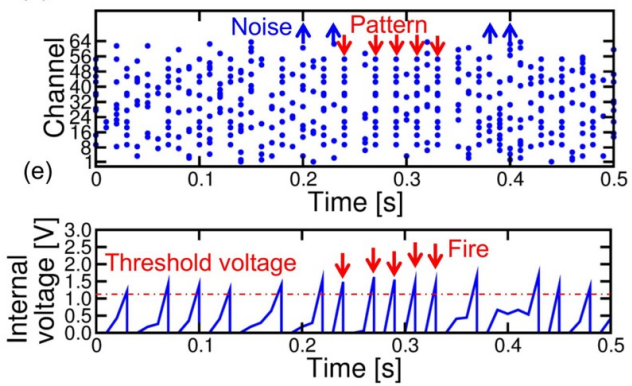

Fig. 8 (a) Schematic representation of a 2-layer feed-forward neural network with 64 PREs fully connected to one POST by 64 1T1R synapses. To enable pattern learning, (b) a pattern and (c) random noisy images are presented to the network with a random sequence as shown by the raster plot in (d). (e) The presentation of the pattern causes POST fires because of $V_{\text {int }}$ exceeding the threshold, thus leading to potentiation in the synapses with $\Delta t>0$. Random noise presentation instead may cause depression, because of stimulation of PRE channels shortly after a fire event with $\Delta t<0$. Reprinted with permission from [83]. Copyright 2016 IEEE.

\section{RRAM networks}

\subsection{Feed-forward networks}

\subsubsection{Simulation results}

The demonstration of the STDP learning rule at the level of individual synapses opens the way for unsupervised learning in full feed-forward networks, such as the 


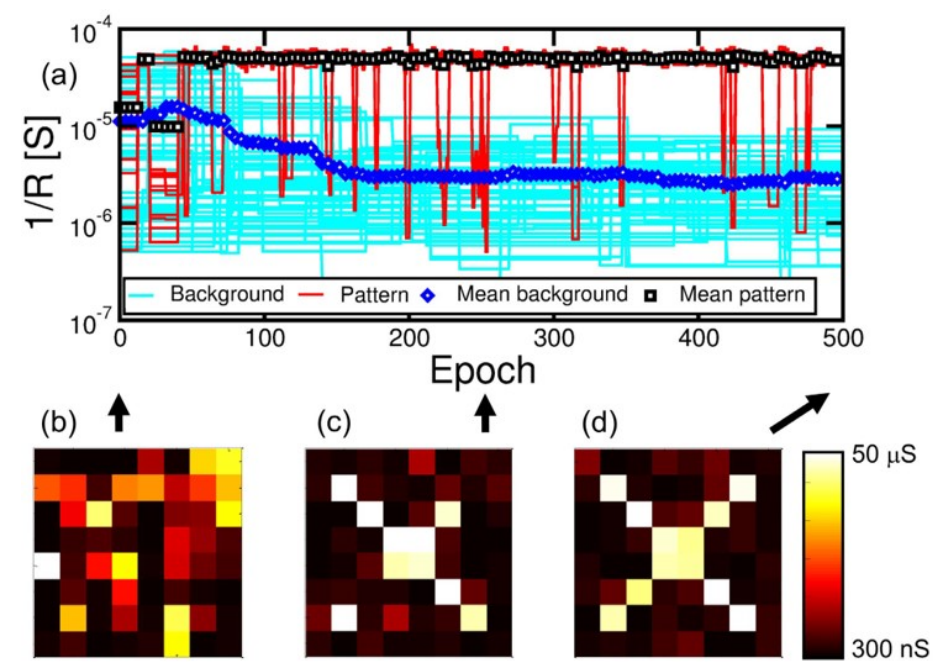

Fig. 9 (a) Calculated synaptic weights in the pattern (red) and background (cyan) as a function of time, obtained by a simulation of unsupervised pattern learning with a RRAM synapse model. (b) Starting from random synaptic states, each lying between HRS and LRS, synapses change their weight according to the stochastic learning process via potentiation of pattern weights and depression of background synapses (c, d). The black and blue traces indicate the average weight of pattern and background synapses, respectively. Reprinted with permission from [83]. Copyright 2016 IEEE.

perceptron network [95] depicted in Fig. 8a, consisting of a 2-layer neural network with 64 PREs and a single POST [83]. In the network, each PRE is connected to the POST via a 1T1R synapse as the one described in Fig. 4. A stochastic learning approach is adopted to induce unsupervised learning, namely, automatic potentiation of synapses within a reference pattern, and depression of all other synapses. The stochastic learning approach consists of the presentation of the reference pattern, e.g. the alphabetical letter $X$ (Fig. 8b). During this presentation, all PREs belonging to the pattern collectively generate a spike which is applied to the corresponding 1T1R synapses. Alternatively, a random noise pattern, e.g., the one shown in Fig. 8c, is presented, each one stimulating random PRE channels. Noise is randomly alternated with the pattern at each epoch, namely, the periodic times marking the submission of a pattern to the network. While the presentation of the pattern causes POST fire, thus potentiation of the active synapses within a certain epoch, the presentation of noise is crucial since it allows for the depression of all the synapses in the background, namely, the space not included in the pattern. This is possible because noise activates random synapses soon after POST fire, thus satisfying the condition for depression with $\Delta t<0$ according to the STDP learning rule.

Fig. 8 also shows a typical sequence of pattern and noise submissions to the input layer (d) and the internal potential $V_{i n t}$ of the POST as a function of time (e). The presentation of the pattern generally causes fire, hence leading to potentiation of the 
active synapses according to STDP. On the other hand, the random synapses activated after POST fire are depressed according to STDP, thus further supporting the importance of the noise alternation to induce depression. Note that the network is never stimulated by two consecutive pattern submissions to avoid activation of the pattern after fire, which could cause unwanted depression of pattern synapses [83]. Fig. 9a shows the calculated evolution of synaptic weights as a function of epochs during training phase, where each epoch corresponds to a time interval of $10 \mathrm{~ms}$. The detailed map of synaptic weights is shown in the color plots of Fig. 9b-d, describing the weight distribution at epochs 0, 250 and 500. Starting from a uniform distribution of synaptic weights at epoch 0 , the pattern synapses undergo a relatively abrupt potentiation within the first 50 epochs. On the other hand, background synapses require a longer time of about 150 epochs for depression, due to the random activation and depression of individual random synapses during the stochastic learning process. The different timescale for potentiation and depression is clearly indicated by the average conductance of pattern and background synapses in Fig. 9a. These simulation results evidence the capability of visual pattern learning according to STDP in a 2-layer perceptron neural network equipped with 1T1R synapses.

\subsubsection{Hardware demonstration of unsupervised learning}

Pattern learning via STDP was demonstrated in hardware via RRAM-based neural networks by achieving remarkable performances on both large [76] and small scale [84, 86, 87, 88]. Fig. 10 shows the reference architecture for unsupervised learning, consisting of a 2-layer perceptron with spiking neurons and 1T1R synapses. This scheme was adopted in a full-hardware implementation with physical RRAM devices and spiking neurons [87].

Fig. 11 shows a schematic illustration of the neural network circuit (a) and the corresponding hardware implementation on a printed circuit board (PCB) (b). The neural network consists of a 2-layer perceptron including 16 PREs, 16 1T1R synapses and a single POST [87, 88]. The PRE spikes were implemented via digital switches enabling the application of a voltage $\mathrm{V}_{G}$ to the gate of the 1T1R synapses. The PRE switches were controlled by an Arduino Due microcontroller $(\mu \mathrm{C})$, which also served as leaky integrate-and-fire (LIF) circuit of the POST for the digital integration of the synaptic currents, which were initially converted into an analog voltage by an external transimpedance amplifier. The feedback spike to the 1T1R synapses at fire was generated by the $\mu \mathrm{C}$, driving a multiplexer (MUX) to provide the appropriate voltage to the TE according to the scheme in Fig. 5.

Fig. 12 shows an experimental demonstration of unsupervised learning of a $4 \times 4$ visual pattern via the hardware spiking neural network in Fig. 11 [87]. The initial weights of the 16 1T1R synapses were prepared in a random state between LRS and HRS, then a diagonal pattern was submitted with stochastic alternation with random noise images. Fig. 12a-d shows the color plots of the synaptic weights during the unsupervised learning process of 1000 epochs $(10 \mathrm{~s})$. Fig. 12e shows the stochastic submission of PRE spikes, representing the pattern or noise consisting of a 3\% of 


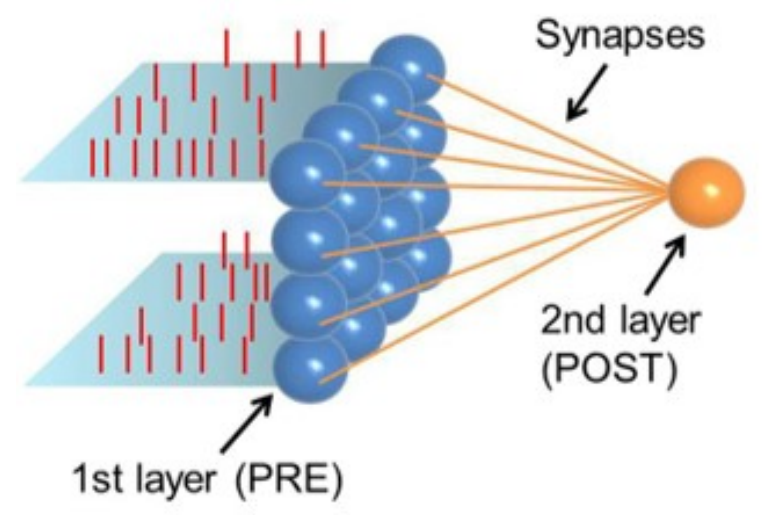

Fig. 10 Illustrative scheme of a 2-layer neural network with perceptron structure including $4 \times 4$ PREs and one POST connected by 16 1T1R synapses. Reprinted from [87], which is licensed under a Creative Commons Attribution 4.0 International License.

(a)

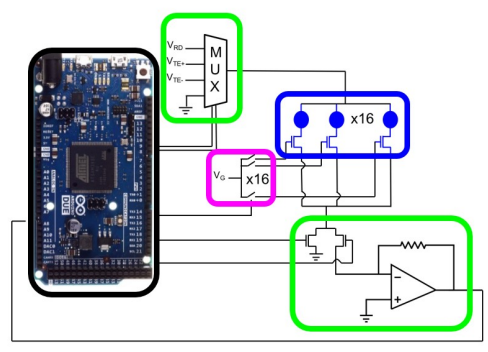

(b)

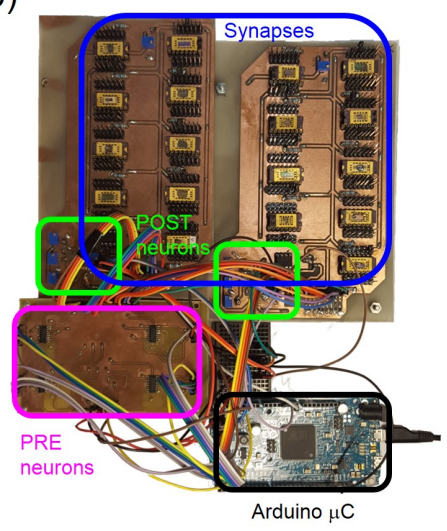

Fig. 11 (a) Circuit schematic and (b) hardware implementation of the RRAM-based feed-forward network depicted in Fig. 10. Reprinted from [87], which is licensed under a Creative Commons Attribution 4.0 International License.

activated channels at each epoch to induce background depression (see Sec. 3). The pattern and noise were presented with equal probabilities of 50\%. The relatively small percentage (3\%) of activated channels in the noise image was adopted to prevent unwanted noise-induced fires which could lead to unstable behavior during the learning process [88]. Fig. 12f shows the detailed evolution of all the pattern (red) and background (blue) synaptic weights evidencing the fast convergence of pattern weights to high conductance values (potentiation) and a more gradual transition of 
(a)

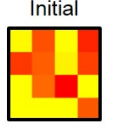

(b)

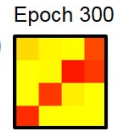

(c)

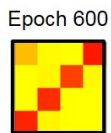

(d)

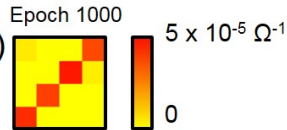

(e)

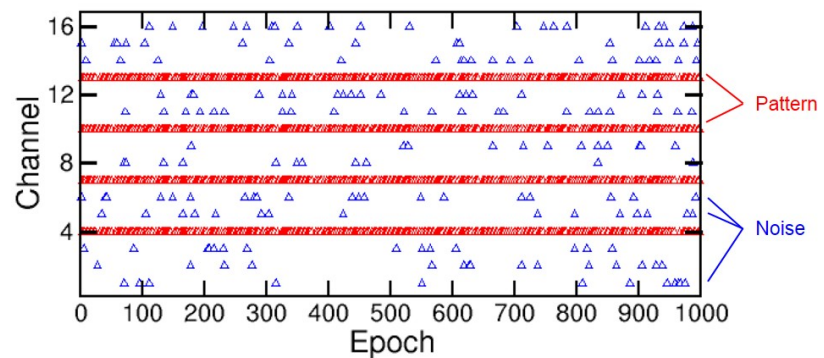

(f)

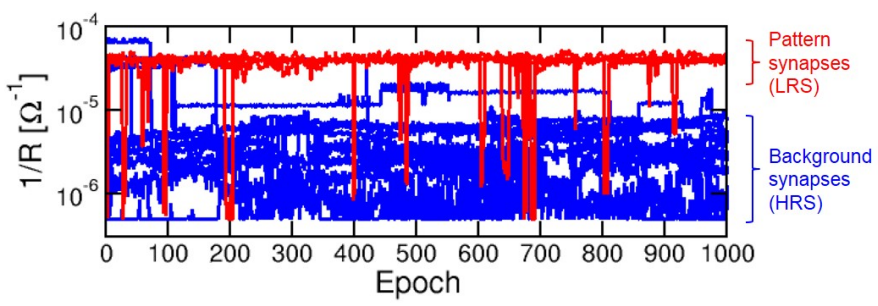

Fig. 12 (a-d) Color plots of the synaptic weights measured during a pattern learning experiment performed by the network of Fig. 11 with a diagonal pattern. (e) Raster plot of PRE spikes within pattern (red) and background (blue) channels. (f) Measured synaptic weights showing the adjustment of pattern synapses to LRS and background synapses to HRS. Reprinted from [87], which is licensed under a Creative Commons Attribution 4.0 International License.

background weights toward low conductance values (depression).

The ability to induce potentiation and depression in real time based on the submitted input spikes allows to quickly adapt the stored weights to a dynamically changing stimulation. To prove the ability to learn dynamic patterns, the network of Fig. 11 was trained with a sequence of 3 distinct patterns, while monitoring the synaptic weights in real time during learning.

Fig. 13a-c shows the 3 patterns which were sequentially presented to the first layer of the neural network during the experiment, while Fig. 13d shows a typical noise image which was alternated with the patterns. After preparing the synaptic weights in HRS (Fig. 13e), the network was externally stimulated by pattern \#1 for 300 epochs ( $3 \mathrm{~s})$, resulting in the potentiation of pattern synapses and depression of background synapses, as evidenced in Fig. 13f. In the following 300 epochs (epochs 301600 ), pattern \#2 was submitted, causing the readjustment of the synaptic weights to adapt to the new pattern, while the previous one was forgotten (Fig. 13g). At epoch 601 , pattern \#3 was presented in the PRE spikes, and eventually learnt by the synaptic network as evidenced by the color plot in Fig. 13h. Fig. 13i shows the raster plot of PRE spikes evidencing the pattern and noise presentation to the network as a 
(a)

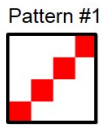

(b)

(e)

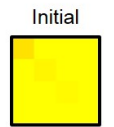

(f)

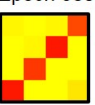

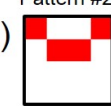

(C)

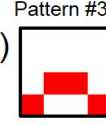

Epoch 300

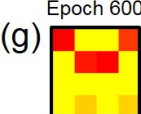

(d)
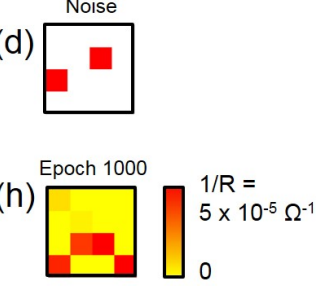

(i)

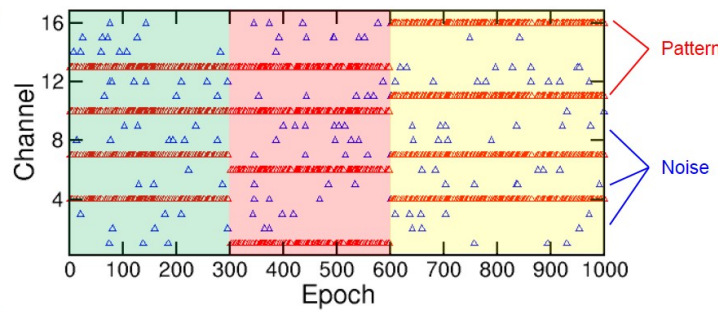

(j)

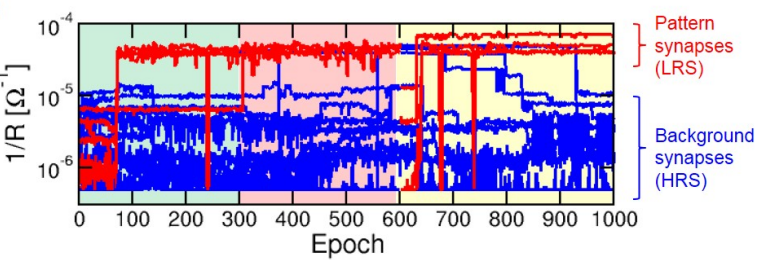

Fig. 13 Experimental demonstration of dynamic pattern learning as a result of sequential application of (a) pattern \#1, (b) pattern \#2, and (c) pattern \#3, each alternated with (d) random noise spikes. (e-h) Color plots of measured synaptic weights during learning experiment which, (e) initialized in a random weight configuration, evidence an effective adaptation to the (f) pattern \#1, (g) pattern \#2, and (h) pattern \#3 within epoch 300, 600 and 1000, respectively. (i) Raster plot of PRE spikes generated as a result of pattern (red) and noise (blue) presentation to the first layer of the neural network and (j) evolution of synaptic weights within the pattern (red) and the background (blue) as a function of epochs, showing a selective potentiation of pattern synapses and a slower depression of background synapses during each training phase. Reprinted from [87], which is licensed under a Creative Commons Attribution 4.0 International License.

function of epochs during each training phase, while Fig. 13j shows the measured synaptic weights as a function of time, further supporting online unsupervised learning by STDP.

A slightly more sophisticated perceptron is displayed in Fig. 14a, featuring 2 POSTs in the second layer for the learning and recognition of 2 distinct patterns. POST1 and POST2 are each connected to the 3x3 PRE layer via 9 1T1R synapses capable of STDP. Also, to avoid learning of the same pattern by the 2 POSTs, the first POST (POST1) and the second POST (POST2) were controlled by the $\mu \mathrm{C}$ to implement a winner-take-all (WTA) learning optimization scheme [74, 96], where the fire of one POST caused the reset of the internal potential in the other POST. This was achieved by 2 inhibitory synapses connecting POST1 to POST2, and POST2 to POST1, to 
(a)

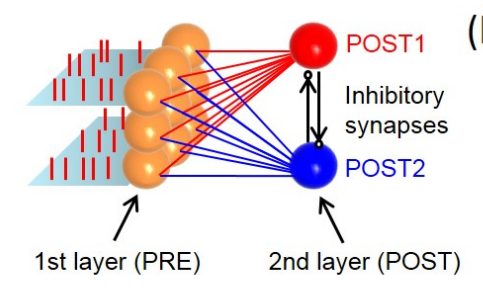

(c)

(d) Epoch: $1000 \quad 2000 \quad 3000 \quad 4000 \quad 5000$

d)

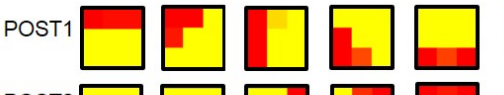

(e)

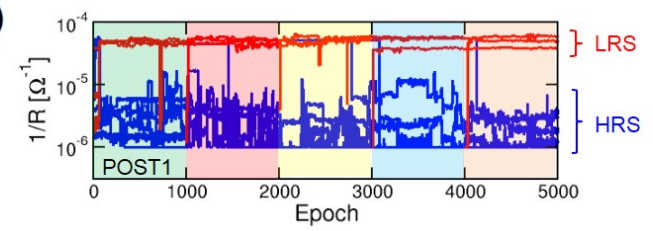

(f)

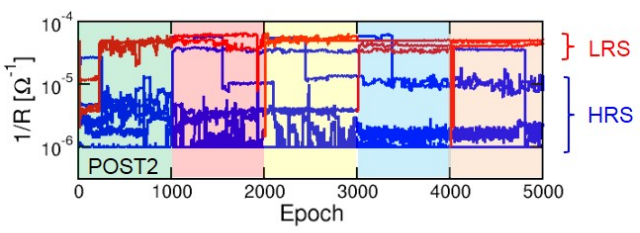

Fig. 14 (a) Sketch of a perceptron network with 2 POSTs in the output neuron layer to implement multiple pattern learning according to the winner-take-all (WTA) scheme. (b) Patterns submitted to the PRE layer, consisting of a top bar and a bottom bar, which were then gradually modified by a counter-clockwise rotation by one step every 1000 epochs to experimentally demonstrate the capability of online dynamic learning. (c, d) Color plots of synaptic weights connecting the input layer to POST1 and POST2, respectively, evidencing the online adaptation to the dynamically changing patterns. (e, f) Measured synaptic weights for POST1 and POST2, respectively, showing the evolution of pattern (red) and background (blue) synaptic weights during the learning process. Note the adjustment of synaptic weights to LRS or HRS, for pattern or background synapses, respectively, in every phase of the online learning experiment. Reprinted from [87], which is licensed under a Creative Commons Attribution 4.0 International License.

allow for bidirectional WTA [74].

In the experiment, the 2 patterns and the usual noise images were submitted to the $3 \times 3$ input layer with random alternated sequence. A top bar and a bottom bar were used as initial patterns for the first 1000 epochs, as shown in Fig. 14b. After this first phase, the bars were modified by a 1-step counterclockwise shift along the perimeter of the $3 \times 3$ frame, until the bar reached the bottom, from the top, or vice versa. Each new learning phase lasted 1000 epochs. Fig. 14c-d shows color plots of the conductance of synapses connected to POST1 and POST2, respectively, during the 
(a)

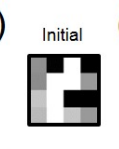

(e)

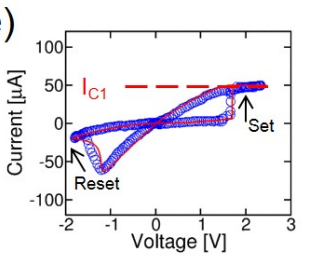

(c)

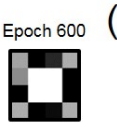

(d)

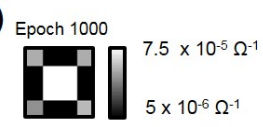

(f)

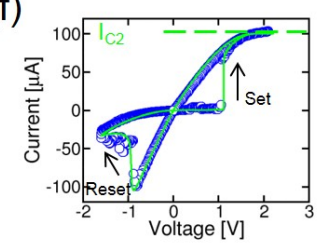

(g)

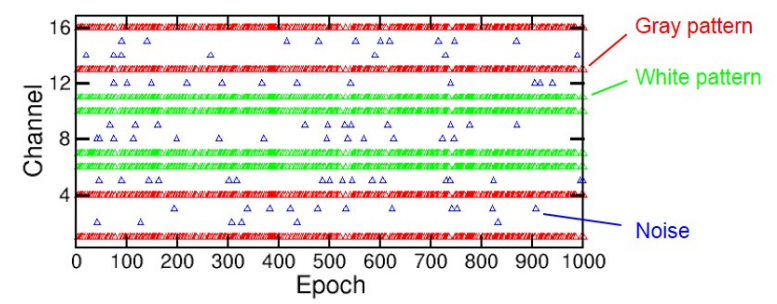

(h)

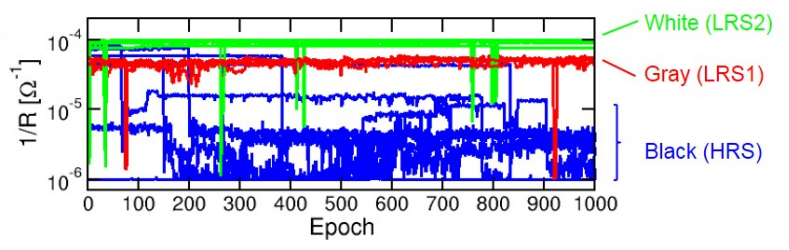

Fig. 15 Color plots of measured synaptic weights at epoch (a) 0, (b) 300, (c) 600, and (d) 1000 as a result of a gray-scale pattern learning. Whereas the black level corresponds to the HRS, gray and white levels correspond to two different low resistance states, namely LRS1 and LRS2, with the conductance of LRS1 being smaller than LRS2. (e, f) Measured and calculated $I-V$ curves for the 1T1R synapses, showing the formation of LRS1 and LRS2, respectively, by the set transition with different gate voltages $\mathrm{V}_{G 1}$ and $\mathrm{V}_{G 2}$, with $V_{G 1}<V_{G 2}$, resulting in compliance currents $I_{C 1}<I_{C 2}$, thus causing LRS1 resistance being higher than LRS2. (g) Raster plot of PRE spikes evidencing the input submission of gray and white patterns with different applied $\mathrm{V}_{G}$, and noise patterns to obtain synaptic depression, hence black level or HRS. (h) Measured synaptic weights during learning, evidencing the adjustment of the conductance to one of the 3 gray levels, namely HRS, LRS1, and LRS2. Reprinted from [87], which is licensed under a Creative Commons Attribution 4.0 International License.

dynamic learning process, evidencing the capability of the 2 POSTs to separately learn the 2 submitted patterns, and to respond to consecutive pattern shifts by gradually adjusting the synaptic weights [87]. Fig. 14e-f shows the synaptic weights of POST1 and POST2, respectively, as a function of epochs during the learning experiment, further evidencing the synaptic plasticity in response to the input dynamic patterns.

All previous examples consider digital input patterns, consisting of $0 / 1$ states for 
each channel, which could be represented by HRS and LRS of the synapse, respectively. On the other hand, a more realistic case is the gray-scale image, where analog stimulation in the first layer, e.g., mapped in the spike frequency or spike amplitude, requires more than 2 resistive levels to represent the input patterns. To this purpose, the gray-scale pattern learning process described in Fig. 15 considers a HRS level, corresponding to the black tone in the figure, and 2 different LRS levels, called LRS1 and LRS2, with the weight of LRS1 being smaller than LRS2 [87]. Fig. 15a-d shows the color plots of the measured synaptic weights during a 1000epoch gray-scale pattern learning experiment performed by the hardware neural network with $3 \times 3$ PREs and one POST. Starting from a random weight configuration, the synaptic weights show the learning of a gray-scale image with 3 levels via a selective analog potentiation process. To obtain these results, LRS1 and LRS2 were achieved by using $1 \mathrm{~T} 1 \mathrm{R}$ synapses with 2 different compliance currents $\mathrm{I}_{C 1}=50 \mu \mathrm{A}$ and $\mathrm{I}_{C 2}=100 \mu \mathrm{A}$, respectively, as a result of the application of the gate voltages $\mathrm{V}_{G 1}=2.1 \mathrm{~V}$ and $\mathrm{V}_{G 2}=2.5 \mathrm{~V}$, as shown by the $I-V$ curves in Fig. 15e-f. The resulting conductance values are LRS1 (gray level) being lower than LRS2 (white level) because of the lower $\mathrm{I}_{C}$ resulting in a higher resistance [63]. Fig. 15g shows the sequence of PRE spikes submitted to the network to implement the 3-level grayscale image learning, while Fig. $15 \mathrm{~h}$ shows the evolution of the synaptic weights with time, indicating the learning of 3 distinct gray-scale levels due to analog potentiation for white and gray tones and noise-induced depression for black tone, respectively. These experimental results support the capability of multi-level pattern learning in 1T1R synapses, paving the way for color-scale image learning.

\subsection{Recurrent neural networks}

\subsubsection{Attractor formation}

While feed-forward networks can be helpful in several applications, such as learning and recognition of different kind of patterns, most of the information processing in the human brain is done by recurrent neural networks (RNNs), where at least one feedback loop exists connecting the output layer to the input layer [97]. For instance, the brain ability to retrieve a previously stored memory, also referred to as attractor state, in response to a partial stimulus, has been the subject of intense studies. According to biological observations, it is believed that this emergent computational ability results from the specific recurrent synaptic topology in interested brain areas such as the hippocampus. Thus, spiking RNNs were modeled and designed leading to both CMOS-based $[98,99,100,101,102,103,104]$ and memristor-based circuit implementations $[105,106,107,108,109,110,111]$. Fig. 16a shows a simplified sketch of a RNN consisting of 4 neurons, each providing both excitatory and in-

hibitory stimulations to each of the other 3 neurons, as well as receiving an external input X. Specifically, according to the well-known Hopfield network topology [112], 
(a)

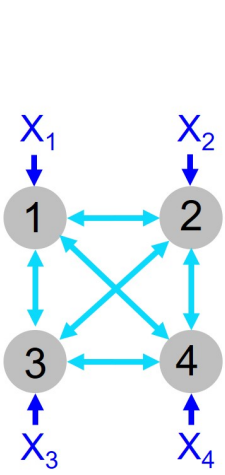

(b)

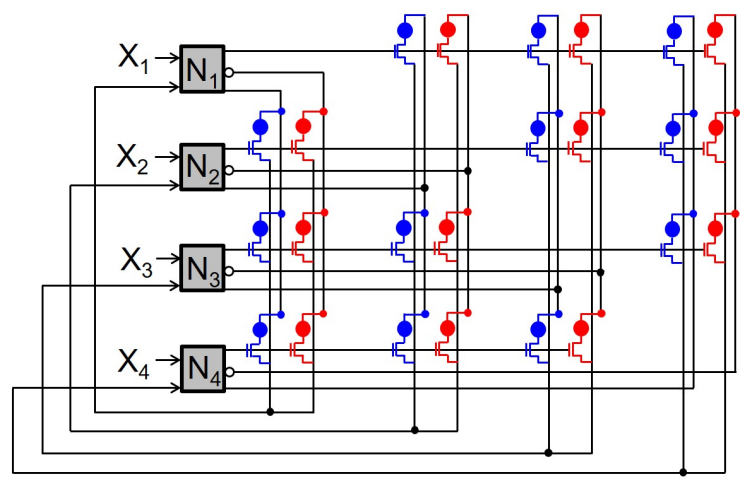

(c)

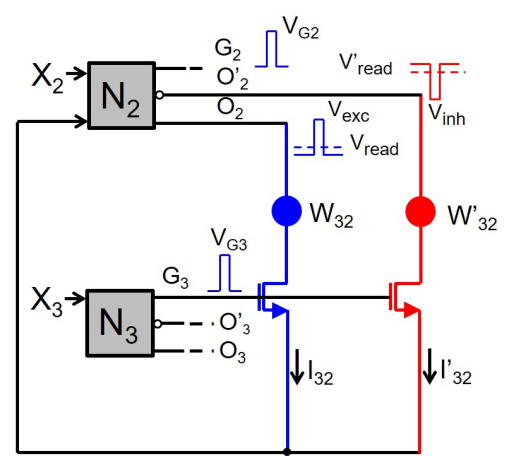

Fig. 16 (a) Illustrative scheme and (b) corresponding circuit implementation of a RNN with the Hopfield network configuration, namely with no self-feedback connections, in the case of 4 fullyconnected neurons. The synapses consist of $1 \mathrm{~T} 1 \mathrm{R}$ structures and are operated as either excitatory or inhibitory connections between 2 neurons in the RNN. (c) Schematic illustration of RNN operation during the training phase at 2 neurons $\left(\mathrm{N}_{2}\right.$ and $\left.\mathrm{N}_{3}\right)$ and their respective synapses. The time overlap between neuron spikes causes potentiation of excitatory synapses, and depression of inhibitory synapses. Adapted with permission from [111]. Copyright 2018 IEEE.

no self-feedback is present in any of the neurons, which prevents a divergent dynamics occurring during network operation.

Fig. 16b shows the circuit implementation of the RNN with spiking neurons and 1T1R synapses [110, 111]. Here, integrate-and-fire neurons are fully connected one to each other by excitatory (blue) and inhibitory (red) $1 \mathrm{~T} 1 \mathrm{R}$ synapses. The generic $i$-th neuron has 2 inputs, namely the external current spike $\mathrm{X}_{i}$, and the total synaptic current activated by other neurons, and 3 outputs, namely (i) $\mathrm{G}_{i}$, which is applied to the gate of 1T1R synapses along the $i$-th row, (ii) $\mathrm{O}_{i}$, which is applied to the TE of excitatory 1T1R synapses along the $i$-th column, and (iii) $\mathrm{O}^{\prime}{ }_{i}$, which is applied to the TE of inhibitory 1T1R synapses along the $i$-th column.

The operation of the RNN during the learning process of an attractor state is de- 
(a)

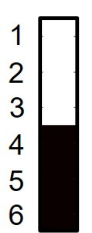

(b)

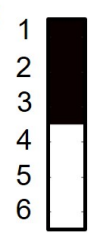

(c)

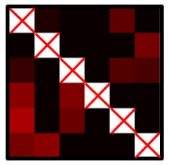

(d)

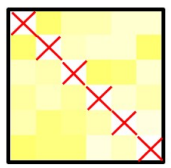

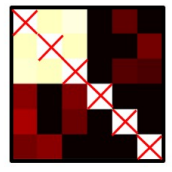

$\mathrm{X}$

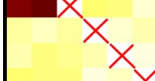

(e)

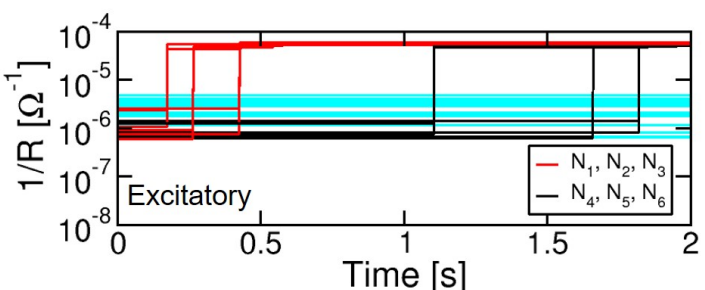

(f)

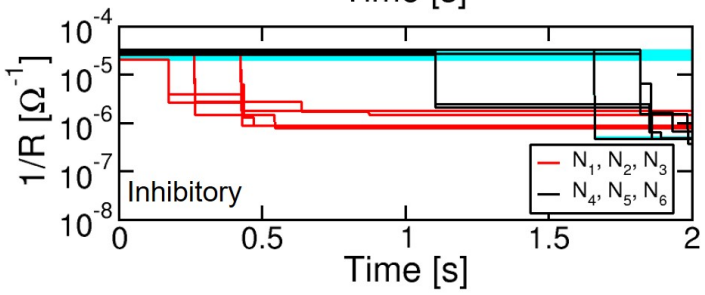

Fig. 17 Simulation of the learning process in a 6-neuron RNN, where two orthogonal attractor states are formed in response to external stimulation of neurons $\mathrm{N}_{1}, \mathrm{~N}_{2}$, and $\mathrm{N}_{3}$ (a), followed by the external stimulation of neurons $\mathrm{N}_{4}, \mathrm{~N}_{5}$, and $\mathrm{N}_{6}$ (b). Color code representation of the conductance values of (c) excitatory and (d) inhibitory synapses at times $0 \mathrm{~s}, 1 \mathrm{~s}$, and $2 \mathrm{~s}$. Weights of the (e) excitatory and (f) inhibitory synapses evidencing the selective potentiation/depression of the excitatory/inhibitory synaptic weights within the attractor activated by stimulation of $\mathrm{N}_{1}, \mathrm{~N}_{2}$, and $\mathrm{N}_{3}$ during the first 1-s-phase, followed by the potentiation/depression of excitatory/inhibitory synapses within the second attractor by stimulation of $\mathrm{N}_{4}, \mathrm{~N}_{5}$, and $\mathrm{N}_{6}$ during the consecutive 1-s-learning phase.

scribed in Fig. 16c for the specific pair of neurons $\mathrm{N}_{2}$ and $\mathrm{N}_{3}$, the excitatory synapse $\mathrm{W}_{32}$ and the inhibitory synapse $\mathrm{W}^{\prime}{ }_{32}$. At the fire event of $\mathrm{N}_{3}$, the gates of all the excitatory/inhibitory synapses along the $3^{\text {rd }}$ row are activated, thus inducing synaptic currents which are proportional to the synaptic weight. The synaptic currents are activated by a relatively small voltage $V_{\text {read }}$ being positive for excitatory synapses, 
and negative for inhibitory synapses. If the total current collected at the input of $\mathrm{N}_{2}$ exceeds the fire threshold, the neuron $\mathrm{N}_{2}$ fires. If the fires of the 2 neurons occur at the same time, both gate and TE pulses are applied to synapses $\mathrm{W}_{32}$ and $\mathrm{W}_{32}$ at the same time, resulting in potentiation of the excitatory synapse, because of the positive TE voltage $V_{\text {exc }}$ causing set transition, and depression of the inhibitory synapse, because of the negative TE voltage $V_{i n h}$ causing reset transition $[110,111]$. Thus, the stimulation by external spikes causes network training according to the Hebbian rule, where 'neurons that fire together wire together' [113]. The potentiation of excitatory synapses and the depression of inhibitory synapses cause the formation of an attractor state in the RNN. Note that the potentiation of excitatory synapses and the depression of inhibitory synapses are simplified cases of the STDP in the 1T1R synapses described in Sec. 3, where the bipolar voltage pulse at the TE is replaced by a unipolar voltage pulse, with either positive voltage $V_{\text {exc }}$ or negative voltage $V_{i n h}$. Fig. 17 shows the simulation results of a sequential learning of two orthogonal attractors, namely 2 attractors with no neurons in common, via a 6-neuron RNN based on the network architecture shown in Fig. 16b [110]. First, all excitatory weights were prepared in HRS, whereas all inhibitory weights were prepared in LRS. Then, the pool of neurons $\mathrm{N}_{1}, \mathrm{~N}_{2}$, and $\mathrm{N}_{3}$ were externally stimulated for $1 \mathrm{~s}$, as shown in Fig. 17a. Finally, external current spikes were applied to another pool including $\mathrm{N}_{4}$, $\mathrm{N}_{5}$, and $\mathrm{N}_{6}$ for the following 1-s-long training phase (Fig. 17b). Fig. 17c shows the evolution of the excitatory synaptic weights, indicating the potentiation of excitatory synapses in the first attractor $\left(\mathrm{N}_{1}, \mathrm{~N}_{2}, \mathrm{~N}_{3}\right)$ during the first learning phase, followed by the potentiation of excitatory synapses in the second attractor $\left(\mathrm{N}_{4}, \mathrm{~N}_{5}, \mathrm{~N}_{6}\right)$ in the second learning phase. Note that external stimulation was asynchronous and had an average frequency of $200 \mathrm{~Hz}$ for stimulated neurons. Similarly, Fig. 17d shows the color plots for inhibitory weights at increasing times, evidencing the depression of inhibitory weights in the attractors. Fig. 17e shows the detailed time evolution of excitatory weights, while Fig. 17f shows inhibitory weights during the two learning phases, further supporting the RNN capability of learning orthogonal attractors.

\subsubsection{Associative memory}

After the attractor learning, the RNN is operated in a different mode aimed at testing or recalling the stored network states $[107,108,110,111]$. A key property of the RNN is that, after attractors are formed in the network, it is possible to recall an attractor even in the presence of a partial or erroneous stimulus of the attractor, which is important for error-tolerant brain-inspired cognitive systems [98, 112]. This type of attractor recall is at the origin of associative learning, namely a fundamental cognitive primitive in the mammalian brain, which received in-depth theoretical and experimental studies, as indicated by the well-known Pavlov's dog experiments [114]. To illustrate the associative learning in the RRAM-based RNN, Fig. 18 shows simulation results for recalling the attractor $\left(\mathrm{N}_{1}, \mathrm{~N}_{2}, \mathrm{~N}_{3}\right)$, and its significance in terms of associative learning according to the Pavlov's dog experiments [110]. If the food presentation to the dog is always combined with the ringing of a bell, the 'bell' and 
(a)

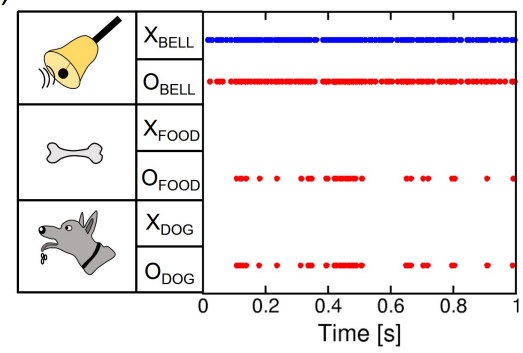

(b)

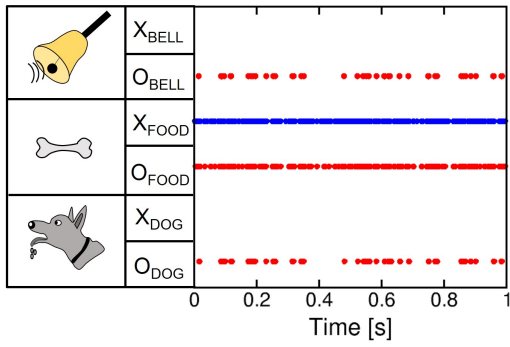

Fig. 18 Illustrative explanation of an associative memory referring to the Pavlov's dog experiments. The regular application of an external stimulus such as a bell's ring during feeding leads to the formation of an attractor state linking bell's ring, food and salivation. As a result, the sound of a bell's ring after training induces the activation of the whole attractor, including the neurons associated with the concept of food, and the stimulus to salivation (a). The attractor is similarly activated by the direct sight of food (b). Reprinted with permission from [110]. Copyright 2017 IEEE.

(a)

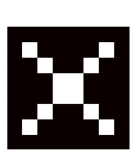

(b)

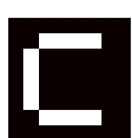

(c)
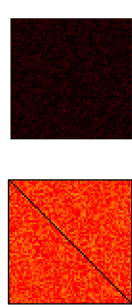

(d)
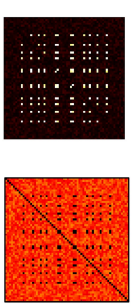

(e)
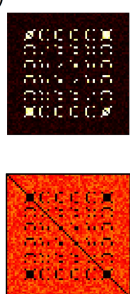

(f)
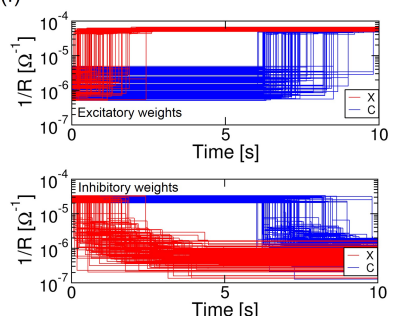

Fig. 19 Simulation of the formation of two attractors as a result of external stimulation of a 64neuron RNN due to the sequential submission of pattern ' $\mathrm{X}$ ' for $5 \mathrm{~s}$ followed by pattern ' $\mathrm{C}$ ' for another 5-s-long phase. ( $\mathrm{a}, \mathrm{b})$ Pattern ' $\mathrm{X}$ ' and ' $\mathrm{C}$ ', respectively. (c-e) Color code representation of the weights of (top) excitatory and (bottom) inhibitory synapses at times $0 \mathrm{~s}, 5 \mathrm{~s}$, and $10 \mathrm{~s}$, starting from HRS and LRS, respectively. (f) Calculated weights of excitatory synapses (top) and inhibitory synapses (bottom), clearly indicating the sequential formation of the attractor ' $\mathrm{X}$ ' (red), followed by the attractor 'C' (blue). Adapted with permission from [111]. Copyright 2018 IEEE.

'food' are associated, i.e., an attractor is formed in the dog's brain. Consequently, whenever the dog hears the bell's ring alone, it resuscitates the concept of food and the stimulus to salivation (Fig. 18a), similar to the direct presentation of food (Fig. 18b).

\subsubsection{Pattern learning and reconstruction}

RNN can provide insight regarding some typical human brain functionalities, such as the memory formation and error-tolerant retrieval [99, 107, 108, 111]. Fig. 19 
(a)

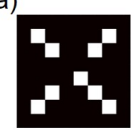

(b)

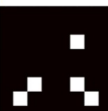

(c)

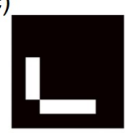

(d)

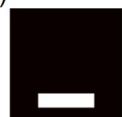

(e)

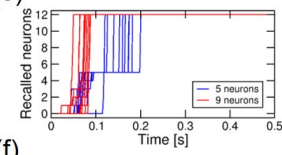

(f)

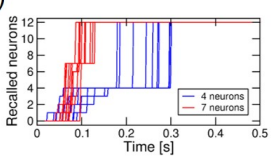

(g)

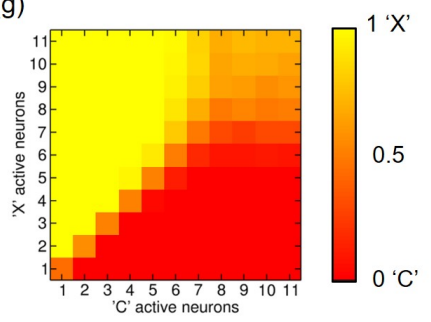

Fig. 20 Incomplete patterns used for simulating pattern reconstruction after pattern learning with (a) 9 active channels and (b) 5 active channels instead of the correct 12, for pattern ' $\mathrm{X}$ '. Incomplete patterns with (c) 7 active channels and (d) 4 active channels instead of the correct 12, for pattern 'C'. Number of activated neurons within (e) the attractor ' $\mathrm{X}$ ' and (f) the attractor ' $\mathrm{C}$ ' as a function of time, for different number of externally-stimulated neurons. (g) Color map of probability $\mathrm{P}$ of reconstructing attractor ' $\mathrm{X}$ ', as a function of the number of externally activated neurons in pattern ' $\mathrm{X}$ ' and pattern ' $\mathrm{C}$ '. The probability of reactivating the attractor ' $\mathrm{C}$ ' can be obtained as 1-P. Adapted with permission from [111]. Copyright 2018 IEEE.

shows simulation results of pattern learning in a RRAM-based RNN with 64 neurons arranged with the architecture of Fig. 16b [111]. Orthogonal attractors were formed by presenting 2 patterns in a sequence, namely the image ' $X$ ' (Fig. 19a) followed by the image 'C' (Fig. 19b), both presented to the RNN for $5 \mathrm{~s}$. The weights were prepared in HRS for excitatory synapses and LRS for inhibitory synapses, as shown in Fig. 19c. Then, the application of pattern ' $X$ ' led to Hebbian modification of the synapses in the first attractor within $5 \mathrm{~s}$ (Fig. 19d). The presentation of the second pattern for the following $5 \mathrm{~s}$ led to the formation of the second attractor (Fig. 19e). The attractor formation is further illustrated in Fig. 19f, showing the calculated weights of the excitatory synapses (top) and inhibitory synapses (bottom) as a function of time. The 2 phases for learning the ' $X$ ' in the first $5 \mathrm{~s}$ and the ' $\mathrm{C}$ ' in the following $5 \mathrm{~s}$ can be clearly seen.

After the attractor formation, the capability of reactivating the whole attractor by submitting only part of the pattern was tested. Fig. 20 shows the input excitation patterns that were submitted in the simulations, consisting of partial versions of the pattern ' $\mathrm{X}$ ' with only (a) 9 active channels or (b) 5 active channels and partial versions of the pattern ' $C$ ' with only (c) 7 active channels or (d) 4 active channels [111]. Fig. 20e shows the simulation results of attractor recall with the partial patterns in (a) and (b), each case being simulated 10 times for statistical significance. The number of activated neurons increases with time during the submission of the partial pattern, eventually activating all the 12 neurons in the original pattern ' $\mathrm{X}$ '. Note that the average time required to retrieve the whole pattern ' $X$ ' decreases as the number of externally stimulated neurons increases, as a result of the higher synaptic current feeding other unstimulated neurons within the selected attractor. Similar to the previous case for pattern ' $\mathrm{X}$ ', the stimulation of a part of attractor ' $\mathrm{C}$ ' leads to the activation of all the 12 neurons in the attractor, as shown in Fig. $20 \mathrm{f}$.

These results support error tolerant pattern recognition, where a pattern is recog- 
nized even in presence of a bare suggestion, or stimulation of only a fraction of the pattern. To explore the limits of the error tolerant recognition, and the possibility of confusion between competing patterns, Fig. 20g shows a color map of the calculated probability $\mathrm{P}$ of recognizing the pattern ' $\mathrm{X}$ ' after externally stimulating the 64-neuron RNN for $1 \mathrm{~s}$. The recognition probability is reported as a function of the number of externally stimulated neurons belonging to ' $\mathrm{X}$ ' or ' $\mathrm{C}$ '. The reported $\mathrm{P}$ is the average over 1000 simulations for each case. Note that all the simulations eventually led to recognition of either ' $\mathrm{X}$ ' or ' $\mathrm{C}$ ', therefore the probability for recognizing $\mathrm{C}$ is given by 1-P [111]. The results indicate that $\mathrm{P}$ increases as the number of stimulated $\mathrm{X}$-neurons increases, and $\mathrm{P}$ decreases as the number of stimulated $\mathrm{C}$ neurons increases. For similar number of $\mathrm{X}$ - and $\mathrm{C}$ - neurons being excited, the color plot shows random behavior of the RNN with $\mathrm{P}$ of about 50\%. Finally, note that as the stimulated $\mathrm{X}$ - and $\mathrm{C}$-neurons within the submitted test pattern are both above $7, \mathrm{P}$ assumes intermediate values since such a high external excitation can activate either attractors with high probability, thus the recall process is mainly controlled by the stochastic Poisson input spike trains used to stimulate the RNN. The results corroborate the feasibility of error-tolerant brain-inspired RNN with RRAM-based 1T1R synapses capable of STDP.

\section{Conclusions}

This chapter provides an overview of the RRAM-based neuromorphic circuits for brain-inspired computing. RRAM devices and architectures might provide a promising technology for scalable, energy-efficient neuromorphic chips, to tackle the challenges of the emergent big data processing and pervasive Internet of Things (IoT). In this scenario, the RRAM device operation, challenges and emerging technologies are reviewed with reference to novel $\mathrm{SiO}_{2} \mathrm{RRAM}$ capable of improved resistance window and stability for multilevel operation in neural networks. Then, RRAM synapses capable of STDP are described, addressing the physical processes and circuit algorithms allowing for time-dependent potentiation and depression.

Finally, SNN architectures capable of pattern learning and other cognitive computing primitives are discussed, covering both feed-forward architectures and braininspired recurrent SNNs. Pattern learning, associative memory, attractor recognition and error tolerant reconstruction of information are shown by the simulation of RRAM-based RNNs. The scenario supports RRAM-based SNN as a promising and attractive technology for low-power and scalable neuromorphic computing. 


\section{Acknowledgements}

This work has received funding from the European Research Council (ERC) under the European Union's Horizon 2020 research and innovation programme (grant agreement no. 648635).

\section{References}

1. Waser, R. and Aono, M.: Nanoionics-based resistive switching memories. Nat. Mater. 6, 833840 (2007). doi: 10.1038/nmat2023

2. Akinaga, H. and Shima, H.: Resistive random access memory (ReRAM) based on metal oxides. Proc. IEEE 98(12), 2237-2251 (2010). doi: 10.1109/JPROC.2010.2070830

3. Wong, H.-S. P., Lee, H.-Y., Yu, S., Chen, Y.-S., Wu, Y., Chen, P.-S., Lee, B., Chen, F.T., and Tsai, M.-J.: Metal-oxide RRAM. Proc. IEEE 100(6), 1951-1970 (2012). doi: 10.1109/JPROC.2012.2190369

4. Yang, J.J., Strukov, D.B. and Stewart, D.R.: Memristive devices for computing. Nat. Nanotechnol. 8, 13-24 (2013). doi: 10.1038/NNANO.2012.240

5. Ielmini, D.: Resistive switching memories based on metal oxides: mechanisms, reliability and scaling. Semicond. Sci. Technol. 31(6), 063002 (2016). doi:10.1088/0268-1242/31/6/063002

6. Hickmott, T.W.: Low frequency negative resistance in thin anodic oxide films. J. Appl. Phys. 33(9), 2669 (1962). Online: https://doi.org/10.1063/1.1702530

7. Gibbons, J.F. and Beadle, W.E.: Switching properties of thin NiO films. Solid-State Electronics 7(11), 785-790 (1964). Online: https://doi.org/10.1016/0038-1101(64)90131-5

8. Simmons, J.G. and Verderber, R.R.: New conduction and reversible memory phenomena in thin insulating films. Proc. Roy. Soc. A. 301, 77-102 (1967). doi: 10.1098/rspa.1967.0191

9. Beck, A., Bednorz, J.G., Gerber, Ch., Rossel, C. and Widber, D.: Reproducible switching effect in thin oxide films for memory applications. Appl. Phys. Lett. 77(1), 139-141 (2000). doi: 10.1063/1.126902

10. Seo, S., Lee, M.J., Seo, D.H., Jeoung, E.J., Suh, D.-S., Joung, Y.S., Yoo, I.K., Hwang, I.R., Kim, S.H., Byun, I.S., Kim, J.-S., Choi, J.S. and Park, B.H.: Reproducible resistance switching in polycrystalline NiO films. Appl. Phys. Lett. 85(23), 5655 (2004). Online: https://doi.org/10.1063/1.1831560

11. Baek, I.G., Lee, M.S., Seo, S., Lee, M.J., Seo, D.H., Suh, D.-S., Park, J.C., Park, S.O., Kim, H.S., Yoo, I.K., Chung, U.-I. and Moon, I.T.: Highly scalable nonvolatile resistive memory using simple binary oxide driven by asymmetric unipolar voltage pulses. in IEDM Tech. Dig. 587-590 (2004). doi: 10.1109/IEDM.2004.1419228

12. Choi, B.J., Jeong, D.S., Kim, S.K., Rohde, C., Choi, S., Oh, J.H., Kim, H.J., Hwang, C.S., Szot, K., Waser, R., Reichenberg, B. and Tiedke, S.: Resistive switching mechanism of $\mathrm{TiO}_{2}$ thin films grown by atomic-layer deposition. J. Appl. Phys. 98(3), 033715 (2005). Online: https://doi.org/10.1063/1.2001146

13. Zahurak, J., Miyata, K., Fischer, M., Balakrishnan, M., Chhajed, S., Wells, D., Li, H., Torsi, A., Lim, J., Korber, M., Nakazawa, K., Mayuzumi, S., Honda, M., Sills, S., Yasuda, S., Calderoni, A., Cook, B., Damarla, G., Tran, H., Wang, B., Cardon, C., Karda, K., Okuno, J., Johnson, A., Kunihiro, T., Sumino, J., Tsukamoto, M., Aratani, K., Ramaswamy, N., Otsuka, W. and Prall, K.: Process integration of a $27 \mathrm{~nm}, 16 \mathrm{~Gb}$ Cu ReRAM. in IEDM Tech. Dig. 140-143 (2014). doi: 10.1109/IEDM.2014.7046994

14. Ueki, M., Takeuchi, K., Yamamoto, T., Tanabe, A., Ikarashi, N., Saitoh, M., Nagumo, T., Sunamura, H., Narihiro, M., Uejima, K., Masuzaki, K., Furutake, N., Saito, S., Yabe, Y., Mitsuiki, A., Takeda, K., Hase, T. and Hayashi, Y.: Low-power embedded ReRAM technology for IoT applications. in Symp. VLSI Tech. Dig. 108-109 (2015). doi: 10.1109/VLSIT.2015.7223640 
15. Chua, L.O.: Memristor - The missing circuit element. IEEE Trans. Circ. Theory 18(5), 507519 (1971). doi: 10.1109/TCT.1971.1083337

16. Strukov, D.B., Snider, G.S., Stewart, D.R. and Williams, R.S.: The missing memristor found. Nature 453 (7191), 80-83 (2008). doi: 10.1038/nature06932

17. LeCun, Y., Bengio, Y. and Hinton, G.: Deep learning. Nature 521, 436-444 (2016). doi: $10.1038 /$ nature 14539

18. LeCun, Y., Bottou, L., Bengio, Y. and Haffner, P.: Gradient-based learning applied to document recognition. Proc. IEEE 86(11), 2278-2324 (1998). doi: 10.1109/5.726791

19. Burr, G.W., Shelby, R.M., Sidler, S., di Nolfo, C., Jang, J., Boybat, I., Shenoy, R.S., Narayanan, P., Virwani, K., Giacometti, E.U., Kurdi, B.N. and Hwang, H.: Experimental demonstration and tolerancing of a large-scale neural network (165 000 synapses) using phase-change memory as the synaptic weight element. IEEE Trans. Electron Devices 62(11), 3498-3507 (2015). doi: 10.1109/TED.2015.2439635

20. Yao, P., Wu, H., Gao, B., Eryilmaz, S.B., Huang, X., Zhang, W., Zhang, Q., Deng, N., Shi, L., Wong, H.-S. P. and Qian, H.: Face classification using electronic synapses. Nat. Commun. 8:15199 (2017). doi: 10.1038/ncomms15199

21. Prezioso, M., Merrikh-Bayat, F., Hoskins, B.D., Adam, G.C., Likharev, K.K. and Strukov, D.B.: Training and operation of an integrated neuromorphic network based on metal-oxide memristors. Nature 521(7550), 61-64 (2015). doi: 10.1038/nature14441

22. Gu, P., Li, B., Yu, S., Cao, Y., Wang, Y. and Yang, H.: Technological exploration of RRAM crossbar array for matrix-vector multiplication. in $20^{\text {th }}$ Asia and South Pacific Design Automation Conference (ASP-DAC), 106-111 (2015). doi: 10.1109/ASPDAC.2015.7058989

23. Ielmini, D., Sharma, D., Lavizzari S. and Lacaita, A.L.: Reliability impact of chalcogenidestructure relaxation in phase change memory (PCM) cells - Part I: Experimental study. IEEE Trans. Electron Devices 56(5), 1070-1077 (2009). doi: 10.1109/TED.2009.2016397

24. Ambrogio, S., Balatti, S., McCaffrey, V., Wang, D. and Ielmini, D.: Noise-induced resistance broadening in resistive switching memory - Part II: Array statistics. IEEE Trans. Electron Devices 62(11), 3812-3819 (2015). doi: 10.1109/TED.2015.2477135

25. Yu, S., Chen, P.-Y., Cao, Y., Xia, L., Wang, Y. and Wu, H.: Scaling-up resistive synaptic arrays for neuro-inspired architecture: challenges and prospect. in IEDM Tech. Dig. 451-454 (2015). doi: 10.1109/IEDM.2015.7409718

26. Jang, J.-W., Park, S., Burr, G.W., Hwang, H. and Jeong, Y.-H.: Optimization of conductance change in $\operatorname{Pr}_{1-x} \mathrm{Ca}_{x} \mathrm{MnO}_{3}$-based synaptic devices for neuromorphic systems. IEEE Electron Device Lett. 36(5), 457-459 (2015). doi: 10.1109/LED.2015.2418342

27. Moon, K., Kwak, M., Park, J., Lee, D. and Hwang, H.: Improved conductance linearity and conductance ratio of 1T2R synapse device for neuromorphic systems. IEEE Electron Device Lett. 38(8), 1023-1026 (2017). doi: 10.1109/LED.2017.2721638

28. Merolla, P.A., Arthur, J.V., Alvarez-Icaza, R., Cassidy, A.S., Sawada, J., Akopyan, F., Jackson, B.L., Imam, N., Guo, C., Nakamura, Y., Brezzo, B., Vo, I., Esser, S.K., Appuswamy, R., Taba, B., Amir, A., Flickner, M.D., Risk, W.P., Manohar, R. and Modha, D.S.: A million spiking-neuron integrated circuit with a scalable communication network and interface. Science 345(6197), 668-673 (2014). doi: 10.1126/science.1254642

29. Lee, J.H., Delbruck, T. and Pfeiffer, M.: Training deep spiking neural networks using backpropagation. Front. Neurosci. 10:508 (2016). doi: 10.3389/fnins.2016.00508

30. Bi, G.-Q. and Poo, M.-M.: Synaptic modifications in cultured hippocampal neurons: dependence on spike timing, synaptic strength, and post synaptic cell type. J. Neurosci. 18(24), 10464-10472 (1998).

31. Wittenberg, G.M. and Wang, S.S.-H.: Malleability of spike-timing-dependent plasticity at the CA3-CA1 synapse. J. Neurosci. 26(24), 6610-6617 (2006). doi: 10.1523/JNEUROSCI.538805.2006

32. Abbott, L.F. and Nelson, S.B.: Synaptic plasticity: taming the beast. Nat. Neurosci. 3(Suppl), 1178-1183 (2000). doi: 10.1038/81453

33. Song, S., Miller, K.D. and Abbott, L.F.: Competitive Hebbian learning through spike-timingdependent synaptic plasticity. Nat. Neurosci. 3(9), 919-926 (2000). doi: 10.1038/78829 
34. Caporale, N. and Dan, Y.: Spike-timing dependent plasticity: A Hebbian learning rule. Annu. Rev. Neurosci. 31, 25-46 (2008). doi: 10.1146/annurev.neuro.31.060407.125639

35. Ielmini, D.: Resistive switching memories. Wiley Encyclopedia Electrical and Electronic Engineering (EEEE), J. Webster (ed.), (2014 John Wiley \& Sons, Inc.). ISBN: 0-471-13946-7

36. Lee, H.Y., Chen, Y.S., Chen, P.S., Gu, P.Y., Hsu, Y.Y., Wang, S.M., Liu, W.H., Tsai, C.H., Sheu, S.S., Chiang, P.C., Lin, W.P., Lin, C.H., Chen, W.S., Chen, F.T., Lien, C.H. and Tsai, M.: Evidence and solution of over-RESET problem for $\mathrm{HfO}_{x}$ based resistive memory with sub-ns switching speed and high endurance. in IEDM Tech. Dig. 460-463 (2010). doi: 10.1109/IEDM.2010.5703395

37. Lee, H.Y., Chen, P.S., Wu, T.Y., Chen, Y.S., Wang, C.C., Tzeng, P.J., Lin, C.H., Chen, F., Lien, C.H. and Tsai, M.-J.: Low power and high speed bipolar switching with a thin reactive Ti buffer layer in robust $\mathrm{HfO}_{2}$ based RRAM. in IEDM Tech. Dig. 297-300 (2008). doi: 10.1109/IEDM.2008.4796677

38. Govoreanu, B., Kar, G.S., Chen, Y.-Y., Paraschiv, V., Kubicek, S., Fantini, A., Radu, I.P., Goux, L., Clima, S., Degraeve, R., Jossart, N., Richard, O., Vandeweyer, T., Seo, K., Hendrickx, P., Pourtois, G., Bender, H., Altimime, L., Wouters, D.J., Kittl, J.A. and Jurczak, M.: $10 \mathrm{x} 10 \mathrm{~nm}^{2} \mathrm{Hf} / \mathrm{HfO}_{x}$ crossbar resistive RAM with excellent performance, reliability and lowenergy operation. in IEDM Tech. Dig. 729-732 (2011). doi: 10.1109/IEDM.2011.6131652

39. Balatti, S., Ambrogio, S., Wang, Z.-Q., Sills, S., Calderoni, A., Ramaswamy, N. and Ielmini, D.: Voltage-controlled cycling endurance of $\mathrm{HfO}_{x}$-based resistive-switching memory. IEEE Trans. Electron Devices 62(10), 3365-3373 (2015). doi: 10.1109/TED.2015.2463104

40. Wang, W., Fujita, S. and Wong, S.S.: RESET mechanism of $\mathrm{TiO}_{x}$ resistance-change memory device. IEEE Electron Device Lett. 30(7), 733-735 (2009). doi: 10.1109/LED.2009.2021001

41. Park, J., Jung, S., Lee, J., Lee, W., Kim, S., Shin, J. and Hwang, H.: Resistive switching characteristics of ultra-thin $\mathrm{TiO}_{x}$. Microelectron. Eng. 88(7), 1136-1139 (2011). doi: 10.1016/j.mee.2011.03.050

42. Lee, M.-J., Lee, C.B., Lee, D., Lee, S.R., Chang, M., Hur, J.H., Kim, Y.-B., Kim, C.-J., Seo, D.H., Seo, S., Chung, U.-I., Yoo, I.-K. and Kim, K.: A fast, high-endurance and scalable non-volatile memory device made from asymmetric $\mathrm{Ta}_{2} \mathrm{O}_{5-x} / \mathrm{TaO}_{2-x}$ bilayer structures. Nat. Mater. 10(8), 625-630 (2011). doi: 10.1038/nmat3070

43. Yang, J.J., Zhang, M.-X., Strachan, J.P., Miao, F., Pickett, M.D., Kelley, R.D., MedeirosRibeiro, G. and Williams, R.S.: High switching endurance in $\mathrm{TaO}_{x}$ memristive devices. Appl. Phys. Lett. 97(23), 232102 (2010). Online: https://doi.org/10.1063/1.3524521

44. Kund, M., Beitel, G., Pinnow, C.-U., Roehr, T., Schumann, J., Symanczyk, R., Ufert, K.-D. and Mueller, G.: Conductive bridging RAM (CBRAM): An emerging non-volatile memory technology scalable to sub $20 \mathrm{~nm}$. in IEDM Tech. Dig. 754-757 (2005). doi: 10.1109/IEDM.2005.1609463

45. Aratani, K., Ohba, K., Mizuguchi, T., Yasuda, S., Shiimoto, T., Tsushima, T., Sone, T., Endo, K., Kouchiyama, A., Sasaki, S., Maesaka, A., Yamada, N. and Narisawa, H.: A novel resistance memory with high scalability and nanosecond switching. in IEDM Tech. Dig. 783-786 (2007). doi: 10.1109/IEDM.2007.4419064

46. Gopalan, C., Ma, Y., Gallo, T., Wang, J., Runnion, E., Saenz, J., Koushan, F., Blanchard, P. and Hollmer, S.: Demonstration of conductive bridging random access memory (CBRAM) in logic CMOS process. Solid State Electron. 58, 54-61 (2011). Online: https://doi.org/10.1016/j.sse.2010.11.024

47. Ambrogio, S., Balatti, S., Choi, S. and Ielmini, D.: Impact of the mechanical stress on switching characteristics of electrochemical resistive memory. Adv. Mater. 26(23), 38853892 (2014). doi: 10.1002/adma.201306250

48. Liu, Q., Sun, J., Lv, H., Long, S., Yin, K., Wan, N., Li, Y., Sun, L. and Liu, M.: Real-time observation on dynamic growth/dissolution of conductive filaments in oxide-electrolyte-based ReRAM. Adv. Mater. 24(14), 1844-1849 (2012). doi: 10.1002/adma.201104104

49. Belmonte, A., Kim, W., Chan, B.T., Heylen, N., Fantini, A., Houssa, M., Jurczak, M. and Goux, L.: A thermally stable and high-performance 90-nm $\mathrm{Al}_{2} \mathrm{O}_{3} / \mathrm{Cu}$-based 1T1R CBRAM cell. IEEE Trans. Electron Devices 60(11), 3690-3695 (2013). doi: 10.1109/TED.2013.2282000 
50. Schindler, C., Weides, M., Kozicki, M.N. and Waser, R.: Low current resistive switching in $\mathrm{CuSiO}_{2}$ cells. Appl. Phys. Lett. 92(12), 122910 (2008). Online: https://doi.org/10.1063/1.2903707

51. Calderoni, A., Sills, S., Cardon, C., Faraoni, E. and Ramaswamy, N.: Engineering ReRAM for high-density applications. Microelectronic Engineering 147, 145-150 (2015). Online: https://doi.org/10.1016/j.mee.2015.04.044

52. Balatti, S., Ambrogio, S., Gilmer, D.C. and Ielmini, D.: Set variability and failure induced by complementary switching in bipolar RRAM. IEEE Electron Device Lett. 34(7), 861-863 (2013). doi: 10.1109/LED.2013.2261451

53. Ambrogio, S., Balatti, S., Cubeta, A., Calderoni, A., Ramaswamy, N. and Ielmini, D.: Statistical fluctuations in $\mathrm{HfO}_{x}$ resistive-switching memory: Part I - Set/Reset variability. IEEE Trans. Electron Devices 61(8), 2912-2919 (2014). doi: 10.1109/TED.2014.2330200

54. Ambrogio, S., Balatti, S., McCaffrey, V., Wang, D. and Ielmini, D.: Noise-induced resistance broadening in resistive switching memory - Part I: Intrinsic cell behavior. IEEE Trans. Electron Devices 62(11), 3805-3811 (2015). doi: 10.1109/TED.2015.2475598

55. Ambrogio, S., Balatti, S., McCaffrey, V., Wang, D. and Ielmini, D.: Noise-induced resistance broadening in resistive switching memory - Part II: Array statistics. IEEE Trans. Electron Devices 62(11), 3812-3819 (2015). doi: 10.1109/TED.2015.2477135

56. Song, J., Woo, J., Prakash, A., Lee, D. and Hwang, H.: Threshold selector with high selectivity and steep slope for cross-point memory array. IEEE Electron Device Lett. 36(7), 681-683 (2015). doi: 10.1109/LED.2015.2430332

57. Chen, W., Barnaby, H.J. and Kozicki, M.N.: Volatile and non-volatile switching in $\mathrm{Cu}-\mathrm{SiO}_{2}$ programmable metallization cells. IEEE Electron Device Lett. 37(5), 580-583 (2016). doi: 10.1109/LED.2016.2540361

58. Bricalli, A., Ambrosi, E., Laudato, M., Maestro, M., Rodriguez, R. and Ielmini, D.: $\mathrm{SiO}_{x^{-}}$ based resistive switching memory (RRAM) for crossbar storage/select elements with high on/off ratio. in IEDM Tech. Dig. 87-90 (2016). doi: 10.1109/IEDM.2016.7838344

59. Wang, Z., Joshi, S., Savel'ev, S.E., Jiang, H., Midya, R., Lin, P., Hu, M., Ge, N., Strachan, J.P., Li, Z., Wu, Q., Barnell, M., Li, G.-L., Xin, H.L., Williams, R.S., Xia, Q. and Yang, J.J.: Memristors with diffusive dynamics as synaptic emulators for neuromorphic computing. Nat. Mater. 16, 101-108 (2017). doi: 10.1038/nmat4756

60. Midya, R., Wang, Z., Zhang, J., Savel'ev, S.E., Li, C., Rao, M., Jang, M.H., Joshi, S., Jiang, H., Lin, P., Norris, K., Ge, N., Wu, Q., Barnell, M., Li, Z., Xin, H.L., Williams, R.S., Xia, Q. and Yang, J.J.: Anatomy of Ag/Hafnia-based selectors with $10^{10}$ nonlinearity. Adv. Mater. 29(12), 1604457 (2017). doi: 10.1002/adma.201604457

61. Bricalli, A., Ambrosi, E., Laudato, M., Maestro, M., Rodriguez, R. and Ielmini, D.: Resistive switching device technology based on silicon oxide for improved on-off ratio - Part II: Select devices. IEEE Trans. Electron Devices (2018). doi: 10.1109/TED.2017.2776085

62. Bricalli, A., Ambrosi, E., Laudato, M., Maestro, M., Rodriguez, R. and Ielmini, D.: Resistive switching device technology based on silicon oxide for improved on-off ratio - Part I: Memory devices. IEEE Trans. Electron Devices (2018). doi: 10.1109/TED.2017.2777986

63. Ielmini, D.: Modeling the universal set/reset characteristics of bipolar RRAM by fieldand temperature-driven filament growth. IEEE Trans. Electron Devices 58(12), 4309-4317 (2011). doi: 10.1109/TED.2011.2167513

64. Wedig, A., Luebben, M., Cho, D.-Y., Moors, M., Skaja, K., Rana, V., Hasegawa, T., Adepalli, K.K., Yildiz, B., Waser, R. and Valov, I.: Nanoscale cation motion in $\mathrm{TaO}_{x}$, $\mathrm{HfO}_{x}$ and $\mathrm{TiO}_{x}$ memristive systems. Nat. Nanotechnol. 11(1), 67-74 (2016). doi: 10.1038/nnano.2015.221

65. Larentis, S., Nardi, F., Balatti, S., Gilmer, D.C. and Ielmini, D: Resistive switching by voltagedriven ion migration in bipolar RRAM - Part II: Modeling. IEEE Trans. Electron Devices 59(9), 2468-2475 (2012). doi: 10.1109/TED.2012.2202320

66. Ambrogio, S., Balatti, S., Gilmer, D.C. and Ielmini, D.: Analytical modeling of oxide-based bipolar resistive memories and complementary resistive switches. IEEE Trans. Electron Devices 61(7), 2378-2386 (2014). doi: 10.1109/TED.2014.2325531 
67. Nardi, F., Larentis, S., Balatti, S., Gilmer, D.C. and Ielmini, D.: Resistive switching by voltage-driven ion migration in bipolar RRAM - Part I: Experimental study. IEEE Trans. Electron Devices 59(9), 2461-2467 (2012). doi: 10.1109/TED.2012.2202319

68. Yu, S., Wu, Y. and Wong, H.-S. P.: Investigating the switching dynamics and multilevel capability of bipolar metal oxide resistive switching memory. Appl. Phys. Lett. 98(10), 103514 (2011). Online: https://doi.org/10.1063/1.3564883

69. Balatti, S., Larentis, S., Gilmer, D.C. and Ielmini, D.: Multiple memory states in resistive switching devices through controlled size and orientation of the conductive filament. Adv. Mater. 25(10), 1474-1478 (2013). doi: 10.1002/adma.201204097

70. Zhao, L., Chen, H.-Y., Wu, S.-C., Jiang, Z., Yu, S., Hou, T.-H., Wong, H.-S. P. and Nishi, Y.: Multi-level control of conductive nano-filament evolution in $\mathrm{HfO}_{2}$ ReRAM by pulse-train operations. Nanoscale 6(11), 5698-5702 (2014). doi: 10.1039/C4NR00500G

71. Prakash, A., Park, J., Song, J., Woo, J., Cha, E.-J. and Hwang, H.: Demonstration of low power 3-bit multilevel cell characteristics in a $\mathrm{TaO}_{x}$-based RRAM by stack engineering. IEEE Electron Device Lett. 36(1), 32-34 (2015). doi: 10.1109/LED.2014.2375200

72. Masquelier, T. and Thorpe, S.J.: Unsupervised learning of visual features through spike timing dependent plasticity. PLoS Comput Biol 3(2):e31 (2007). doi: 10.1371/journal.pcbi.0030031

73. Masquelier, T., Guyonneau, R. and Thorpe, S.J.: Spike timing dependent plasticity finds the start of repeating patterns in continuous spike trains. PLose One, 3(1):e1377 (2008). doi: 10.1371/journal.pone.0001377

74. Masquelier, T., Guyonneau, R. and Thorpe, S.J.: Competitive STDP-based spike pattern learning. Neural Computation 21(5), 1259-1276 (2009). doi: 10.1162/neco.2008.06-08-804

75. Suri, M., Bichler, O., Querlioz, D., Cueto, O., Perniola, L., Sousa, V., Vuillaume, D., Gamrat, C. and DeSalvo, B.: Phase change memory as synapse for ultra-dense neuromorphic systems: application to complex visual pattern extraction. in IEDM Tech. Dig. 79-82 (2011). doi: 10.1109/IEDM.2011.6131488

76. Zamarreno-Ramos, C., Camunas-Mesa, L.A., Perez-Carrasco, J.A., Masquelier, T., SerranoGotarredona, T. and Linares-Barranco, B.: On spike-timing-dependent-plasticity, memristive devices, and building a self-learning visual cortex. Front. Neurosci. 5:26 (2011). doi: 10.3389/fnins.2011.00026

77. Bichler, O., Suri, M., Querlioz, D., Vuillaume, D., DeSalvo, B. and Gamrat, C.: Visual pattern extraction using energy-efficient "2-PCM synapse" neuromorphic architecture. IEEE Trans. Electron Devices 59(8), 2206-2214 (2012). doi: 10.1109/TED.2012.2197951

78. Suri, M., Bichler, O., Querlioz, D., Palma, G., Vianello, E., Vuillaume, D., Gamrat, C. and DeSalvo, B.: CBRAM devices as binary synapses for low-power stochastic neuromorphic systems: auditory (cochlea) and visual (retina) cognitive processing applications. in IEDM Tech. Dig. 235-238 (2012). doi: 10.1109/IEDM.2012.6479017

79. Yu, S., Gao, B., Fang, Z., Yu, H., Kang, J. and Wong, H.-S. P.: A neuromorphic visual system using RRAM synaptic devices with sub-pJ energy and tolerance to variability: experimental characterization and large-scale modeling. in IEDM Tech. Dig. 239-242 (2012). doi: 10.1109/IEDM.2012.6479018

80. Park, S., Sheri, A., Kim, J., Noh, J., Jang, J., Jeon, M., Lee, B., Lee, B.R., Lee, B.H. and Hwang, H.: Neuromorphic speech systems using advanced ReRAM-based synapse. in IEDM Tech. Dig. 625-628 (2013). doi: 10.1109/IEDM.2013.6724692

81. Serrano-Gotarredona, T., Masquelier, T., Prodromakis, T., Indiveri, G. and Linares-Barranco, B.: STDP and STDP variations with memristors for spiking neuromorphic learning systems. Front. Neurosci. 7:2 (2013). Online: https://doi.org/10.3389/fnins.2013.00002

82. Ambrogio, S., Ciocchini, N., Laudato, M., Milo, V., Pirovano, A., Fantini P. and Ielmini, D.: Unsupervised learning by spike timing dependent plasticity in phase change memory (PCM) synapses. Front. Neurosci. 10:(56) (2016). doi: 10.3389/fnins.2016.00056

83. Ambrogio, S., Balatti, S., Milo, V., Carboni, R., Wang, Z., Calderoni, A., Ramaswamy, N. and Ielmini, D.: Neuromorphic learning and recognition with one-transistor-one-resistor synapses and bistable metal oxide RRAM. IEEE Trans. Electron Devices 63(4), 1508-1515 (2016). doi: 10.1109/TED.2016.2526647 
84. Serb, A., Bill, J., Khiat, A., Berdan, R., Legenstein, R. and Prodromakis, T.: Unsupervised learning in probabilistic neural networks with multi-state metal-oxide memristive synapses. Nat. Commun. 7:12611 (2016). doi: 10.1038/ncomms12611

85. Covi, E., Brivio, S., Serb, A., Prodromakis, T., Fanciulli, M. and Spiga, S.: Analog memristive synapse in spiking networks implementing unsupervised learning. Front. Neurosci. 10:482 (2016). doi: 10.3389/fnins.2016.00482

86. Milo, V., Pedretti, G., Carboni, R., Calderoni, A., Ramaswamy, N., Ambrogio, S. and Ielmini, D.: Demonstration of hybrid CMOS/RRAM neural networks with spike time/rate-dependent plasticity. in IEDM Tech. Dig., 440-443 (2016). doi: 10.1109/IEDM.2016.7838435

87. Pedretti, G., Milo, V., Ambrogio, S., Carboni, R., Bianchi, S., Calderoni, A., Ramaswamy, N., Spinelli, A.S. and Ielmini, D.: Memristive neural network for on-line learning and tracking with brain-inspired spike timing dependent plasticity. Sci. Rep. 7(1), 5288 (2017). doi: 10.1038/s41598-017-05480-0

88. Pedretti, G., Milo, V., Ambrogio, S., Carboni, R., Bianchi, S., Calderoni, A., Ramaswamy, N., Spinelli, A.S. and Ielmini, D.: Stochastic learning in neuromorphic hardware via spike timing dependent plasticity with RRAM synapses. IEEE Journal on Emerging and Selected Topics in Circuits and Systems (JETCAS), 8(1), pp. 77-85 (2018). doi: 10.1109/JETCAS.2017.2773124

89. Diehl, P.U. and Cook, M.: Unsupervised learning of digit recognition using spike-timingdependent plasticity. Front. Comput. Neurosci. 9:99 (2015). doi: 10.3389/fncom.2015.00099

90. Ambrogio, S., Balatti, S., Milo, V., Carboni, R., Wang, Z., Calderoni, A., Ramaswamy, N. and Ielmini, D.: Novel RRAM-enabled 1T1R synapse capable of low-power STDP via burstmode communication and real-time unsupervised machine learning. in IEEE Symposium on VLSI Technology, 196-197 (2016). doi: 10.1109/VLSIT.2016.7573432

91. Ielmini, D., Ambrogio, S., Milo, V., Balatti, S. and Wang, Z.-Q.: Neuromorphic computing with hybrid memristive/CMOS synapses for real-time learning. IEEE Int. Symp. on Circuits and Systems (ISCAS), pp. 1386-1389 (2016). doi:10.1109/ISCAS.2016.7527508

92. McCulloch, W.S. and Pitts, W.H.: A logical calculus of the ideas immanent in nervous activity. Bull. Math Biophys. 5, 115-133 (1943).

93. Prezioso, M., Merrikh-Bayat, F., Hoskins, B., Likharev, K. and Strukov, D.: Self-adaptive spike-time-dependent plasticity of metal-oxide memristors. Sci. Rep. 6:21331 (2016). doi: $10.1038 /$ srep21331

94. Wang, Z., Ambrogio, S., Balatti, S. and Ielmini, D.: A 2-transistor/1-resistor artificial synapse capable of communication and stochastic learning in neuromorphic systems. Front. Neurosci. 8:438 (2015). doi:10.3389/fnins.2014.00438

95. Rosenblatt, F.: The perceptron: a probabilistic model for information storage and organization in the brain. Psychological review 65(6), 386-408 (1958).

96. Maass, W.: On the computational power of winner-take-all. Neural Comput. 12(11), 25192535 (2000).

97. Grossberg, S.: Recurrent neural networks. Scholarpedia, 8(2):1888 (2013). doi: $10.4249 /$ scholarpedia. 1888

98. Indiveri, G. and Liu, S.-C.: Memory and information processing in neuromorphic systems. Proc. IEEE 103(8), 1379-1397 (2015). doi: 10.1109/JPROC.2015.2444094

99. Giulioni, M., Corradi, F., Dante, V. and Del Giudice, P.: Real time unsupervised learning of visual stimuli in neuromorphic VLSI systems. Sci. Rep. 5:14730 (2015). doi: 10.1038/srep14730

100. Chicca, E., Badoni, D., Dante, V., D’Andreagiovanni, M., Salina, G., Carota, L., Fusi, S. and Del Giudice, P.: A VLSI recurrent network of integrate-and-fire neurons connected by plastic synapses with long-term memory. IEEE Trans. Neural Netw. 14(5), 1297-1307 (2003). doi: 10.1109/TNN.2003.816367.

101. Chicca, E., Stefanini, F., Bartolozzi, C. and Indiveri, G.: Neuromorphic electronic circuits for building autonomous cognitive systems. Proc. IEEE 102(9), 1367-1388 (2014). doi: 10.1109/JPROC.2014.2313954 
102. Neftci, E., Binas, J., Rutishauser, U., Chicca, E., Indiveri, G. and Douglas. R.J.: Synthesizing cognition in neuromorphic electronic systems. Proc. Natl. Acad. Sci. USA (PNAS) 110(37), E3468-E3476 (2013). doi: 10.1073/pnas.1212083110

103. Qiao, N., Mostafa, H., Corradi, F., Osswald, M., Stefanini, F., Sumislawska, D. and Indiveri, G.: A reconfigurable on-line learning spiking neuromorphic processor comprising 256 neurons and 128K synapses. Front. Neurosci. 9:141 (2015). doi: 10.3389/fnins.2015.00141

104. Guo, X., Merrikh-Bayat, F., Gao, L., Hoskins, B.D., Alibart, F., Linares-Barranco, B., Theogarajan, L., Teuscher, C. and Strukov, D.B.: Modeling and experimental demonstration of a Hopfield network analog-to-digital converter with hybrid CMOS/memristor circuits. Front. Neurosci. 9:488 (2015). doi: 10.3389/fnins.2015.00488

105. Pershin, Y.V. and di Ventra, M.: Experimental demonstration of associative memory with memristive neural networks. Neural Netw. 23(7), 881-886 (2010). Online: https://doi.org/10.1016/j.neunet.2010.05.001

106. Ziegler, M., Soni, R., Patelczyk, T., Ignatov, M., Bartsch, T., Meuffels, P., Kolhstedt, H.: An electronic version of Pavlov's dog. Adv. Funct. Mater. 22(13), 2744-2749 (2012). doi: 10.1002/adfm.201200244

107. Kuzum, D., Jeyasingh, R.G.D., Yu, S. and Wong, H.-S. P.: Low-energy robust neuromorphic computation using synaptic devices. IEEE Trans. Electron Devices 59(12), 3489-3494 (2012). doi: 10.1109/TED.2012.2217146

108. Eryilmaz, S.B., Kuzum, D., Jeyasingh, R., Kim, S.B., BrightSky, M., Lam, C. and Wong, H.-S. P.: Brain-like associative learning using a nanoscale non-volatile phase change synaptic device array. Front. Neurosci. 8:205 (2014). doi: 10.3389/fnins. 2014.00205

109. Hu, S.G., Liu, Y., Liu, Z., Chen, T.P., Wang, J.J., Yu, Q., Deng, L.J., Yin, Y. and Hosaka, S.: Associative memory realized by a reconfigurable memristive Hopfield neural network. Nat. Commun. 6:7522 (2015). doi: 10.1038/ncomms8522

110. Milo, V., Ielmini, D. and Chicca, E.: Attractor networks and associative memories with STDP learning in RRAM synapses. in IEDM Tech. Dig., 263-266 (2017). doi: 10.1109/IEDM.2017.8268369

111. Milo, V., Chicca, E. and Ielmini, D.: Brain-inspired recurrent neural network with plastic RRAM synapses. IEEE International Symposium on Circuits and Systems (ISCAS), pp. 1-5 (2018). doi: 10.1109/ISCAS.2018.8351523

112. Hopfield, J.J.: Neural networks and physical systems with emergent collective computational abilities. Proc. Natl. Acad. Sci. USA (PNAS), 79, 2554-2558 (1982).

113. Hebb, D. O.: The organization of behavior: a neurophysiological theory. New York: Wiley \& Sons, (1949).

114. Pavlov, I.P.: Conditioned reflexes: An investigation of the physiological activity of the cerebral cortex. Oxford University Press, London (1927). 